\title{
VARIETIES OF DISTRIBUTIVE LATTICES WITH UNARY OPERATIONS I
}

\author{
H. A. PRIESTLEY \\ (Received 29 January 1996; revised 21 October 1996)
}

Communicated by B. A. Davey

\begin{abstract}
A unified study is undertaken of finitely generated varieties $\mathrm{MSP}(\underline{P})$ of distributive lattices with unary operations, extending work of Cornish. The generating algebra $\underline{P}$ is assumed to be of the form $\left(P ; \wedge, \vee, 0,1,\left\{f_{\mu}\right\}\right)$, where each $f_{\mu}$ is an endomorphism or dual endomorphism of $(P ; \wedge, \vee, 0,1)$, and the Priestley dual of this lattice is an ordered semigroup $\mathbf{N}$ whose elements act by left multiplication to give the maps dual to the operations $f_{\mu}$. Duality theory is fully developed within this framework, into which fit many varieties arising in algebraic logic. Conditions on $\mathbf{N}$ are given for the natural and Priestley dualities for $\mathbb{H S P}(\boldsymbol{P})$ to be essentially the same, so that, inter alia, coproducts in $\mathbb{H} \mathbb{S P}(\boldsymbol{P})$ are enriched D-coproducts.
\end{abstract}

1991 Mathematics subject classification (Amer. Math. Soc.): primary 06D05; secondary 06D25, 03G20, $08 \mathrm{~B} 99$.

Keywords and phrases: Priestley duality, natural duality, free algebra, monoid.

\section{Introduction}

This is the first of a series of papers developing the duality theory of varieties of distributive lattices with additional unary operations. Particular varieties of this type first attracted attention as algebraic models in non-classical propositional logic (Kleene and de Morgan algebras, for example). They have since been studied extensively as algebraic structures of interest in their own right, as indicated for example by the numerous papers by Blyth, Varlet and others on Ockham algebras (the simple case in which we have a single unary negation operator satisfying de Morgan's laws). Furthermore, these varieties have provided instructive examples through which the theory of natural dualities has been much advanced.

Natural duality theory has been most extensively developed for classes $\mathscr{A}$ of the

(C) 1997 Australian Mathematical Society 0263-6115/97 \$A2.00+0.00 
form $\mathbb{S P}(P)$, where $\underline{P}$ is a finite algebra. Surveys of this theory can be found in $[15,26,10]$. It was shown in [17] that the basic theory extends to classes $\mathbb{U S P}(\underline{\Pi})$, where $\underline{\Pi}$ is a finite set of finite algebras of the same type. This generalised theory allows us to work with a variety rather than a quasivariety even where these do not coincide, and at no more cost than greater notational complexity. However, as a concession to readers not yet familiar with the theory in its full generality, we restrict attention in this paper to classes expressible as $\operatorname{ISP}(P)$, deferring to [31] the more general cases $\mathbb{D S P}(\underline{\Pi)}$ ) and $\mathbb{H S P}(\underline{\Pi})$, for a finite set $\underline{\Pi}$ of algebras. Even when $\mathscr{A}:=\mathbb{M S P}(\underline{P}) \supsetneqq \mathbb{N S \mathbb { P }}(\underline{P})$, study of the quasivariety $\mathbb{\mathbb { S }} \mathbb{P}(\underline{P})$ suffices for the determination of free algebras in $\mathscr{A}$.

A systematic study of distributive lattices with additional unary operations each of which is either an endomorphism or dual endomorphism was undertaken some years ago by Cornish in [11, 12]. These works provide the setting for our investigations and the starting point for them. Under Priestley duality, the algebraic operations correspond to maps on the dual spaces, which are order-preserving or order-reversing depending on whether the operation is an endomorphism or a dual endomorphism. Cornish exhibited many examples in which the dual maps are given by the continuous action of a monoid, whose elements are tagged with ${ }^{+}$(corresponding to an endomorphism) and ${ }^{-}$(dual endomorphism). He then went on to develop a general theory of \pm -monoids to encompass these examples, within a lattice framework in [11] and in a much wider and more categorical setting in [12].

Cornish considers principally varieties which are generated by a finite algebra whose underlying lattice is Boolean, treating other varieties of interest as subvarieties of these. We elect to consider many such subvarieties in their own right. Given such a variety, the Priestley dual, $N$, of its generating algebra, $\underline{P}$, is a finite poset, acted on by a set of self-maps. We show that in many important cases these maps on $N$ are indexed by the points of $N$, and given by a semigroup action of $N$ on itself. The elements of $N$ are tagged ${ }^{+}$and ${ }^{-}$, to indicate the type of algebraic operations on $\underline{P}$ to which they correspond; to each \pm -element there are two associated fundamental operations, one endomorphism and one dual endomorphism, and these are linked by a pair of identities (see Theorem 2.6). We call such structures $\mathbf{N}=\left(N ; \cdot, e, N^{+}, N^{-}, \leqslant\right)$weak ordered \pm -semigroups (the formal definition is given in Section 2). They extend Cornish's \pm -monoids in three ways:

(i) a partial order replaces Cornish's tacit discrete order;

(ii) a right identity, rather than an identity, is postulated;

(iii) the sets $\mathrm{N}^{+}$and $\mathrm{N}^{-}$are not required to be disjoint.

Reversing the process, we can show that every finite weak ordered \pm -semigroup $\mathbf{N}$ gives rise to a variety $\mathscr{A}^{\mathrm{N}}$, generated by the algebra $\underline{P}$ dual to $\mathbf{N}$. We reveal that $\mathbf{N}$ determines in a very direct way a Priestley duality and a natural duality for $\mathscr{A}^{\mathrm{N}}$, 
and thence the free algebras and coproducts in $\mathscr{A}^{\mathrm{N}}$. Our work was motivated by, and subsumes, the work on Ockham algebra varieties in $[22,17,25,1]$ and (almost) that on Łukasiewicz algebras in [28].

In this paper we set up the general framework and present examples. These include varieties in the following overlapping classes:

- varieties generated by finite subdirectly irreducible Ockham algebras (Stone algebras, Kleene algebras, de Morgan algebras, MS-algebras, ... );

- double MS-algebras, double Stone algebras, linked weak double Stone algebras [34];

- regular $\alpha$-de Morgan algebras [9], involutive Stone algebras [9], Kleene-Stone algebras [23];

- 3-valued Łukasiewicz algebras, and, with an extension of the definitions, $n$-valued Łukasiewicz algebras.

If we are given one of these classes as a variety $\mathbb{H I S P}(\underline{P})$, for some finite algebra $\underline{P}$, then we can immediately fit it into our general scheme. If, alternatively, it is given equationally, then to encompass it we need to draw on information already available in the literature: we require the subdirectly irreducible algebras, and a restricted Priestley duality. In certain cases we also have to switch to a different, but equivalent, set of operations to bring the given variety under our umbrella. However, once our variety has been recognised as a variety $\mathscr{A}^{\mathbf{N}}$, we can instantly describe its free algebras, which may have previously been obtained by algebraic or Priestley duality methods, or may be hitherto unknown. We stress that our method treats all varieties of the type $\mathscr{A}^{\mathbf{N}}$ in a uniform way. A significant merit of working at the level of generality that we do is that results which originally looked specific to individual varieties can be re-cast in a form which reveals that they stem directly from the structure of the semigroups which determine the varieties. Compare, for example, Lemma 4.4 below with Lemma 3.4 in [17]. We note that, although Cornish mentions many of the varieties above, he does not treat them intrinsically. His work also pre-dates the development of the theory of natural dualities as it applies to these varieties, so that this topic is not covered at all in [12]. We are able to show that the condition that $\mathbf{N}$ be a monoid in which right multiplication is always order-preserving is exactly that required to ensure that $\mathscr{A}^{\mathrm{N}}$ has natural and Priestley dualities with especially nice properties in relation to coproducts and free algebras; see Theorem 4.13. Our later papers $[31,29,30]$ are concerned with dualities and identities. In [31] we look at natural dualities in more detail and show how to derive natural dualities for arbitrary subvarieties, and thereby how to obtain equational bases for them. This extends to the setting of the present paper the work carried out for Ockham varieties in [1] and [27], and for individual varieties of double MS-algebras in [33]. In [29] we reveal an algorithmic relationship between 
the identities satisfied by a finite algebra and the axiomatic theories of the structured Priestley spaces of algebras in the generated variety. In [31] and in [30] we focus on one example as an illustration: the variety DMS of double MS-algebras. Here we have two related but non-commuting Ockham negation operators. The natural duality for DMS is based on a 5-element schizophrenic object, which is small enough to analyse with ease, yet is sufficiently complex to show up through its subvarieties important features of the theory which do not emerge in examples previously studied. Three chapters of Blyth and Varlet's book Ockham algebras [6] are devoted to DMS and its subvarieties, using algebraic methods and a little Priestley duality. We obtain comprehensive results on the natural dualities, and are able in [29] to amplify and elucidate Blyth and Varlet's results in a significant way. However our principal reason for choosing this example for detailed study is its suitability for illustrating the general theory.

The author would like to acknowledge the assistance of Raquel Santos in the preparation of this paper. In particular, her detailed work on piggyback dualities for DMS subvarieties in [33] helped lead the way to a number of the general results presented here.

\section{Varieties associated with semigroups}

This section is essentially a synthesis and refinement of results of Cornish in [11] and [12] concerning the action of monoids on distributive lattices and their dual spaces. Many specialisations of these results are well known, notably for varieties of Ockham algebras.

We first establish our conventions regarding the action of semigroups. Let $(\mathrm{N} ; \cdot)$ be a semigroup and let $S$ be a set. A left action of the semigroup $N$ on $S$ is a map $(\mu, y) \mapsto{ }^{\mu} y$ for which ${ }^{\lambda}\left({ }^{\mu} y\right)={ }^{\lambda \mu} y$ for all $y \in S$ and $\lambda, \mu \in N$. Likewise, a right action of the semigroup $N$ on $S$ is a map $(\mu, y) \mapsto y^{\mu}$ such that $\left(y^{\lambda}\right)^{\mu}=y^{\mu \lambda}$. On $N$ itself, the left and right multiplications define left and right actions:

$$
\begin{aligned}
& (\mu, \nu) \mapsto{ }^{\mu} v:=\mu \cdot \nu, \\
& (\mu, \nu) \mapsto v^{\mu}:=v \cdot \mu .
\end{aligned}
$$

We shall wish to take as our fundamental object a semigroup $N$ with the action of left multiplication. The objects of our dual category, $\mathscr{Y}$, will be spaces with a left $N$-action, and $N$ itself will be a member of $\mathscr{Y}$. We shall write $g_{\mu}(y)$ for ${ }^{\mu} y(y \in Y$, $Y \in \mathscr{Y}$ ), so the maps $g_{\mu}$ compose according to the rule $g_{\lambda \mu}=g_{\lambda} \circ g_{\mu}$. The operations of the corresponding algebraic category should then be given by a right action, since Priestley duality is set up by contravariant functors. This is not unnatural, since unary operations are frequently written to the right of their arguments: $a^{+}, a^{\circ}$ for the 
operations of a DMS-algebra, for example. Notwithstanding, we shall often write $f_{\mu}(a)$ for $a^{\mu}$, remembering that $f_{\lambda \mu}=f_{\mu} \circ f_{\lambda}$. These conventions give a familiar look to the results of this section and the next, and to the theory in [29]. We stress that Cornish elects to use categories with the semigroup multiplication and actions reversed, so that, for example, we have left ideals where Cornish has right ideals, and below we write $\mathbf{D}-\mathbf{N}$ where Cornish would write $N-B D$.

We now briefly recall Priestley duality as it applies to categories of distributive lattices with additional operations. Let $\mathscr{A}$ be a class of algebras (not necessarily a variety) whose members are algebras $\underline{A}=(A ; \wedge, \vee, 0,1, F)$ where $(A ; \wedge, \vee, 0,1)$ belongs to the variety $\mathbf{D}$ of bounded distributive lattices and $F$ is a set, indexed by a fixed set (later a semigroup), $N$, of unary operations each of which defines either an endomorphism or a dual endomorphism of $(A ; \wedge, \vee, 0,1)$. Throughout, we shall only be concerned with bounded lattices, and maps between lattices will always be assumed to preserve these bounds. We shall usually write $A$ rather than $\underline{A}$, where the context makes it clear whether we are referring to the algebra, to its D-reduct or to its underlying set. Indeed in places it is hard to maintain an entirely consistent usage without undue pedantry. We retain underlining when we need to distinguish the different personæ of a schizophrenic object, and when it seems helpful for emphasis.

There is a dual category equivalence between $\mathbf{D}$, qua category, and the category $\mathbf{P}$ of Priestley spaces (compact totally order-disconnected spaces); see, for example, [15] or [26] for the duality as it is formulated here, or [18] for a more elementary account. The duality is given by hom-functors $H: \mathbf{D} \rightarrow \mathbf{P}$ (hom-ing into the 2-element chain $\underline{\mathbf{2}}$ in $\mathbf{D}$ ) and $K: \mathbf{P} \rightarrow \mathbf{D}$ (hom-ing into the discretely topologised 2-element chain 2 in $\mathbf{P}$ ), so that

$$
\begin{array}{lll}
H(A):=\mathbf{D}(A, \underline{2}) & \text { and } \quad K(Y):=\mathbf{P}(Y, \underset{2}{2}), \\
H(f):=-\circ f & \text { and } \quad K(\varphi):=-\circ \varphi .
\end{array}
$$

Where we need to make explicit the order, $\leqslant$, and topology, $\mathscr{T}$, of the dual space $H(A)$ of $A$ then we write $(H(A) ; \mathscr{T}, \leqslant)$. At the object level, the equivalence means that, given $A \in \mathbf{D}$, we have $A \cong K H(A)$, where the isomorphism $k_{A}$ is given by evaluation. It is common to suppress $k_{A}$ and to identify $A$ with $K H(A)$, so writing synonymously $a(y)$ and $y(a)(a \in A, y \in H(A))$. We shall frequently do this.

Morphisms in $\mathbf{D}$ correspond (contravariantly) to continuous order-preserving maps in P. Note that the order dual, $A^{d}$, of $A$ has dual space $(H(A), \mathscr{T}, \geqslant)$, where $(H(A) ; \mathscr{T}, \leqslant)$ is the dual space of $A$. Therefore dual homomorphisms between objects in $\mathbf{D}$ correspond to continuous order-reversing maps between the dual objects in $\mathbf{P}$.

Now assume $\mathscr{A}$ is a class of algebras of the same type, each with a $\mathbf{D}$-reduct. The D-reduct of $\mathscr{A}$ is dually equivalent to a full subcategory of $\mathbf{P}$. We afforce the objects 
of this subcategory with continuous order-preserving and order-reversing maps to correspond to the algebraic operations in $\mathscr{A}$, and restrict the morphisms appropriately. We thereby obtain a dual equivalence between $\mathscr{A}$ and a category $\mathscr{Y}^{\mathscr{A}}$ of structured Priestley spaces; we again denote the functors setting this up by $H$ and $K$. We then have the following theorem, originally obtained in [11] and given in [12, Section 6.3].

THEOREM 2.1. Let $N=N^{+} \cup N^{-}$be a fixed set. Let $\mathscr{A}$ be a class of distributivelattice-ordered algebras $\left(A ; \wedge, \vee, 0,1, F_{N}\right)$, where $F_{N}=\left\{f_{\mu} \mid \mu \in N\right\}$ is such that $f_{\mu}$ defines an endomorphism for each $\mu \in N^{+}$and $f_{\mu}$ defines a dual endomorphism for each $\mu \in N^{-}$. Then there is a duality, set up by (the restrictions of) $H$ and $K$, between $\mathscr{A}$ and a category $\mathscr{Y}^{\circ}$ whose objects are of the form $\left(Y ; G_{N}\right)$, where $Y$ is a Priestley space and $G_{N}=\left\{g_{\mu} \mid \mu \in N\right\}$ defines a set of continuous maps from $Y$ to $Y$ such that $g_{\mu}$ is order-preserving (order-reversing) if $f_{\mu}$ is an endomorphism (dual endomorphism). The morphisms of $\mathscr{Y}^{\mathscr{A}}$ are the continuous order-preserving maps commuting with the maps $g_{\mu}$, for $\mu \in N$. Furthermore, for $A \in \mathscr{A}$,

$$
f_{\mu}(a)(x)= \begin{cases}a\left(g_{\mu}(x)\right) & \text { for } \mu \in N^{+}, \\ 1-a\left(g_{\mu}(x)\right) & \text { for } \mu \in N^{-},\end{cases}
$$

for all $a \in A$ and $x \in H(A)$.

In the cases in which we shall be interested the set of operations $F_{N}$ on the algebras in $\mathscr{A}$ can be chosen (generally redundantly) in such a way that the maps $g_{\mu}$ on any given dual space $H(A)$ form a monoid, or just a semigroup, under functional composition. Cornish $[12,5.1]$ defines a \pm -monoid to be a structure $\mathbf{N}=\left(N ; \cdot, e, N^{+}, N^{-}\right)$such that

(M1) $(N ; \cdot, e)$ is a monoid;

(M2) $N=N^{+} \cup N^{-}$;

(M3) for $\sigma, \tau \in\{ \pm\}, N^{\sigma} \cdot N^{\tau} \subseteq N^{\sigma \tau}$;

(M4) $N^{+} \cap N^{-}=\emptyset$.

Here the multiplication on $\{+,-\}$ is the expected one, so that, for example, $N^{+} \cdot N^{-} \subseteq$ $N^{-}$. If (M4) is omitted, then $\mathbf{N}=\left(N ; \cdot, e, N^{+}, N^{-}\right)$is said to be a weak \pm -monoid $([12,5.9])$. We define a (weak) \pm -semigroup analogously, assuming now merely that $e$ is a (distinguished) right identity. Note that $e$ must belong to $N^{+}$, by (M3). The reason for insisting that there be a right identity will be seen in Section 3 .

Given a \pm -semigroup $\mathbf{N}$ we can associate a class $\mathbf{D}-\mathbf{N}$ of algebras $\left(A ; \wedge, \vee, 0,1, F_{N}\right)$ such that $(A ; \wedge, \vee, 0,1)$ is a distributive lattice with 0,1 and the fundamental operations $F_{N}=\left\{f_{\mu} \mid \mu \in N\right\}$ of $A$ are indexed by $N$ in such a way that $f_{\mu}$ is an endomorphism if $\mu \in N^{+}$and a dual endomorphism if $\mu \in N^{-}$. Additionally we require that $f_{\lambda} \circ f_{\mu}=f_{\mu \lambda}$ for all $\lambda, \mu \in N$ and that $f_{e}=$ id (if $N$ is a monoid). In a similar way we define the category $\mathbf{N}-\mathbf{P}$ consisting of structures which are Priestley 
spaces on which a semigroup of maps $g_{\mu}(\mu \in N)$ acts on the left in a continuous manner, with $g_{\lambda} \circ g_{\mu}=g_{\lambda \mu}$ and with $g_{\mu}$ order-preserving if $\mu \in N^{+}$and order-reversing if $\mu \in N^{-}$. Morphisms in the two categories are morphisms of the $\mathbf{D}$ - and $\mathbf{P}$-reducts which preserve the semigroup action.

THEOREM 2.2 (cf. [12, 6.3.6]). For any \pm -semigroup $\mathbf{N}$ the functors $H$ and $K$ set up a dual equivalence between $\mathbf{D}-\mathbf{N}$ and $\mathbf{N}-\mathbf{P}$.

As an example, we cast the well-known restricted Priestley duality for Ockham algebras in its monoid form. Recall that the variety $\mathbf{O}$ of Ockham algebras consists of algebras $(A ; \wedge, \vee, 0,1, \sim)$ where $(A ; \wedge, \vee, 0,1) \in \mathbf{D}$ and where, for all $a, b$ in $A$, $\sim(a \wedge b)=\sim a \vee \sim b, \quad \sim(a \vee b)=\sim a \wedge \sim b, \quad \sim 0=1, \quad \sim 1=0$.

The dual category $\mathscr{Y}^{\mathbf{O}}$ (of Ockham spaces) consists of spaces $(Y ; g)$ where $Y \in \mathbf{P}$ and $g$ is a continuous order-reversing map, with morphisms all continuous orderpreserving maps commuting with $g$. Here $\sim a$ on $A$ and $g$ on $H(A)$ are linked by $(\sim a)(x)=1-a(g(x))$ for all $a \in A, x \in H(A)$.

We can alternatively (but equivalently) regard each $A \in \mathbf{O}$ as being equipped not with the single operation $\sim$ but with the family of operations $\left\{\sim^{k} \mid k=0,1,2, \ldots\right\}$. In the corresponding description of the dual category, the objects would be the spaces $\left(Y ;\left\{g^{k} \mid k=0,1, \ldots\right\}\right)$, where $Y$ and $g$ are as above. We now see that the variety of Ockham algebras may be identified with $\mathbf{D}$-N, where $N=\left\{g^{k} \mid k=0,1,2, \ldots\right\}$ is the monoid freely generated by $g$, and

$$
N^{+}=\left\{g^{2 n} \mid n=0,1, \ldots\right\}, \quad N^{-}=\left\{g^{2 n+1} \mid n=0,1, \ldots\right\} .
$$

We shall henceforth regard $\mathbf{O}$ and $\mathscr{Y}^{\mathbf{o}}$ as being in their revamped, monoid-oriented, forms with an infinite family of operations.

We remark that the duality theory that we develop below in the context of finite semigroups does extend under certain circumstances to varieties associated with infinite semigroups. This happens for $\mathbf{O}$, as the analysis in $[22,20]$ indicates. However, such varieties are rare, and topological considerations intrude. Therefore we restrict henceforth to finite semigroups in this paper.

Suppose $\mathscr{A} \subseteq \mathbf{D}-\mathbf{N}$ and $\mathscr{Y}^{\mathscr{A}} \subseteq \mathbf{N}-\mathbf{P}$ are dual categories as in Theorem 2.1. Then identities satisfied in the class $\mathscr{A}$ will correspond to certain inequality restrictions on the maps $\left\{g_{\mu} \mid \mu \in N\right\}$. In [29] we show that this translation process can be carried out in a uniform and algorithmic way. The forms of identity we wish to consider at this stage are covered by the theorem below. For more in the same vein, see [12, Theorem 6.4.5].

THEOREM 2.3 (from [12, Theorem 6.4.5]). Let $\mathscr{A}$ and $\mathscr{Y}^{\mathscr{A}}$ be as in Theorem 2.1. Let $\lambda, \mu \in N$. 
(i) If $\lambda, \mu$ both belong to $N^{+}$or both belong to $N^{-}$then $f_{\lambda}(a)=f_{\mu}(a)$ holds identically in $\mathscr{A}$ if and only if $g_{\lambda}=g_{\mu}$ holds on all spaces in $\mathscr{Y}^{\mathscr{A}}$.

(ii) If $\mu \in N^{+}$and $\lambda \in N^{-}$then $f_{\mu}(a) \wedge f_{\lambda}(a)=0$ and $f_{\mu}(a) \vee f_{\lambda}(a)=1$ hold identically in $\mathscr{A}$ if and only if $g_{\lambda}=g_{\mu}$ holds on all spaces in $\mathscr{Y}^{\mathscr{A}}$.

We deal principally with finite algebras. An algebra $P$ with a D-reduct is finite if and only if its dual $H(P)$ is finite, and carries the discrete topology (which we always denote by $\tau$ ). Also, any finite partially ordered set becomes an element of $\mathbf{P}$ if it is given the discrete topology; we shall assume finite sets to carry this topology where the context presupposes a topology. Note further that any map defined on a finite object in $\mathbf{P}$ is automatically continuous.

We now come to the core of our approach. We are interested in varieties (or more generally quasivarieties) which are finitely generated. We therefore have a class $\mathscr{A}=\mathbb{H} \mathbb{S} \mathbb{P}(\underline{P})$ or $\mathscr{A}=\mathbb{U S P}(\underline{P})$, where $\underline{P}$ is a finite non-trivial algebra, and whose Priestley dual space, $H(P)$, is a finite poset with the discrete topology, equipped with a family of order-preserving and order-reversing maps. Suppose that the operations $\left\{f_{\mu}\right\}$ of $\underline{P}$ are indexed by the points of $N:=H(P)$, and let $g_{\mu}$ be the map dual to $f_{\mu}$, as in Theorem 2.1. We shall shortly look at some examples. In each of these, $N$ becomes a \pm -monoid when monoid multiplication $\cdot$ is defined by

$$
\mu \nu:=g_{\mu}(\nu) \quad(\mu, \nu \in N)
$$

and elements of $N$ are assigned to $N^{+}$and $N^{-}$as follows:

$$
\begin{aligned}
& \mu \in N^{+} \text {if and only if } f_{\mu} \text { is an endomorphism, } \\
& \mu \in N^{-} \text {if and only if } f_{\mu} \text { is a dual endomorphism. }
\end{aligned}
$$

(In a non-trivial algebra $f_{\mu}$ cannot be simultaneously an endomorphism and a dual endomorphism.) Further, the order and the multiplication on $N$ satisfy

(M5) for all $\mu, v_{1}, v_{2} \in N$,

$$
\nu_{1} \leqslant v_{2} \text { implies } \begin{cases}\mu v_{1} \leqslant \mu v_{2} & \text { if } \mu \in N^{+}, \\ \mu \nu_{1} \geqslant \mu v_{2} & \text { if } \mu \in N^{-} .\end{cases}
$$

We shall say that the structure $\mathbf{N}=\left(N ; \cdot, e, N^{+}, N^{-}, \leqslant\right)$is a (weak) ordered \pmsemigroup if $\left(N ; \cdot, e, N^{+}, N^{-}\right)$is a (weak) \pm -semigroup and the multiplication and order are linked by (M5). We denote the class of all finite ordered \pm -semigroups by $\mathscr{M}$, and all finite weak ordered \pm -semigroups by $\mathscr{N}$. We shall subsume Cornish's theory within ours by regarding any \pm -monoid in his sense as being equipped with the discrete order, so making it an ordered \pm -monoid in our sense. We may also obtain from $\mathbf{N} \in \mathscr{N}$ a weak \pm -semigroup $\mathbf{N}_{\text {= }}$ by forgetting the order. 
Given any $\mathbf{N} \in \mathscr{M}$ (or more generally $\mathbf{N} \in \mathscr{N}$ satisfying (M4)) we have, as indicated above, an algebra we shall henceforth denote by $\underline{P}^{\mathrm{N}}$ (dropping the superscript where $\mathbf{N}$ is understood). We shall see below (Proposition 3.5) that when $\mathbf{N}$ is a discretely ordered \pm -monoid we have $\mathbb{H} \mathbb{S} \mathbb{P}\left(\underline{P}^{\mathbf{N}}\right)=\mathbf{D}$-N. So, extending our earlier notation, we let $\mathbf{D}-\mathbf{N}:=\mathbb{H} \mathbb{S}\left(\underline{P}^{\mathbf{N}}\right)$, and let $\mathbf{N}-\mathbf{P}$ be the dual category. The latter contains $\left(N ;\left\{g_{\mu}\right\}_{\mu \in N}\right)$, which, by an abuse of notation, we shall denote by $\mathbf{N}$. Where D-N is some familiar variety $\mathscr{A}$ we shall sometimes write $\mathbf{N}^{\mathscr{A}}$ rather than $\mathbf{N}$. Note that the same algebra $\underline{P}$ can arise as $\underline{P}^{\mathrm{N}}$ for several different structures $\mathrm{N}$, for two reasons. We have specified a distinguished right identity $e$ in our semigroup. In general, there will be more than one choice; see 2.8(4) for an example. Also, having specified $N$ as a weak \pm -semigroup, there may be different ways to impose the order. The variety $\mathbf{K}$ of Kleene algebras gives the simplest example; see 2.4(1).

We now turn to the promised examples. Here, and in many examples we present subsequently, the $\mathbf{N}_{=}$versions of the monoids $\mathbf{N} \in \mathscr{M}$ appear in [12]. In diagrams of weak ordered \pm -semigroups we indicate the subsets $N^{+}$and $N^{-}$by ${ }^{ \pm}$tags against the points.

2.4. Examples. (1) de Morgan, Kleene and MS-algebras. The variety $M$ of de Morgan algebras is the subvariety of $\mathbf{O}$ consisting of algebras satisfying $\sim^{2} a=a$. The corresponding dual category $\mathscr{Y}^{\mathrm{M}}$ consists of those Ockham spaces $(Y ; g)$ for which $g^{2}=$ id. The variety $\mathbf{M}$ is $\mathbb{\mathbb { S } P}(\underline{M})=\mathbb{M S P}(\underline{M})$, where $\underline{M}$ is the Boolean lattice $\{0, a, b, 1\}$, on which $\sim$ is defined to fix $a$ and $b$ and interchange 0 and 1 . The dual of $\underline{M}$ may be identified with the discretely ordered \pm -monoid $N=\{1, g\}$ in which $g^{2}=1$, and $\mathbf{M}=\mathbf{D}$-N. See [12, 4.11 and 6.3].

The variety $\mathbf{K}$ of Kleene algebras is the subvariety of $\mathbf{M}$ consisting of algebras satisfying $a \wedge \sim a \leqslant b \vee \sim b$. The dual category was first identified by Cornish and Fowler in [13] as the full subcategory of $\mathscr{Y}^{\mathbf{0}}$ consisting of spaces $(Y ; g)$ such that $g^{2}=1$ and such that $Y=\{x \in Y \mid x \leqslant g(x)\} \cup\{x \in Y \mid x \geqslant g(x)\}$. The variety $\mathbf{K}$ is generated (via $\triangle \mathbb{S P}$ or DASP) by $\underline{K}$, the 3-element chain $0<a<1$ with $\sim a=a$. The dual $H(\underline{K})$ can be thought of as the ordered \pm -monoid $\mathbf{N}^{\mathbf{K}}$, as shown in Figure 1(a). Alternatively we may switch the two points, placing 1 at the top.

In a similar manner, the variety of MS-algebras (cf. Cornish's 'polar algebras', [12, 6.3]) is derived from the ordered monoid $\mathbf{N}^{\mathrm{MS}}$ shown in Figure 1(b). The associated discretely ordered monoid $\mathbf{N}_{=}^{\mathrm{MS}}$ gives rise to the Ockham variety $\mathbf{P}_{3,1}\left(=\mathbf{K}_{1,1}\right)$; see 2.5 .

(2) Double MS-algebras. The variety DMS of double MS-algebras consists of algebras $\left(A ; \wedge, \vee, 0,1,^{\circ},+\right)$ such that $\left(A ; \wedge, \vee, 0,1,^{\circ}\right)$ is an MS-algebra, $\left(A ; \wedge, \vee, 0,1,{ }^{+}\right)$is a dual MS-algebra, and the identities $a^{\circ \circ}=a^{\circ+}$ and $a^{++}=a^{+\circ}$ hold. The variety DMS of double MS-algebras is identifiable with D-N, where the associated ordered \pm - monoid has two generators, and is the monoid $M(\alpha, \beta)$ of $[12$, 


$$
\begin{aligned}
& +\jmath g \\
& -\delta 1=g^{2}
\end{aligned}
$$

(a) $\mathbf{N}^{K}$

$$
+\oint_{+} g^{2} \quad-\circ g=g^{3}
$$

(b) $\mathbf{N}^{\mathrm{MS}}$

FIGURE 1

5.7], (with $g, h$ replacing $\alpha, \beta$ ), and ordered as shown in Figure 2. Specifically,

$$
N=\left\{1, g, g^{2}, h, h^{2}\right\} \quad \text { where } \quad g h=g^{2}, h g=h^{2}, g^{3}=g, h^{3}=h .
$$

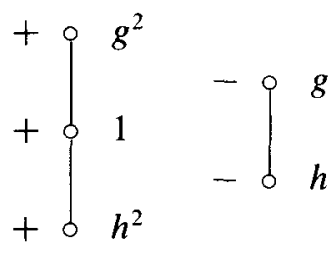

FIGURE 2. $\mathbf{N}^{\text {DMS }}$

Now let us take as our starting point an arbitrary finite ordered semigroup $(N ; \leqslant)$, with right identity $e$. Define maps $g_{\mu}: N \rightarrow N$ by left multiplication: $g_{\mu}(\nu):=$ $\mu \nu$. We should like to form an algebra $\underline{P}=\left(P ; \wedge, \vee, 0,1,\left\{f_{\mu}\right\}_{\mu \in N}\right)$ whose dual is $\left(N ;\left\{g_{\mu}\right\}\right)$. To do this we wish to assign the elements of $N$ to disjoint sets $N^{+}$and $N^{-}$ so that $g_{\mu}$ is order-preserving for $\mu \in N^{+}$and order-reversing for $\mu \in N^{-}$, and then to link $f_{\mu}$ and $g_{\mu}$ according to the formulæ in Theorem 2.1. Define

$$
\begin{aligned}
& N^{++}:=\left\{\mu \in N \mid g_{\mu} \text { is order-preserving }\right\}, \\
& N^{--}:=\left\{\mu \in N \mid g_{\mu} \text { is order-reversing }\right\}
\end{aligned}
$$

and try to define $N^{+}=N^{++}$and $N^{-}=N^{--}$. Provided $N^{++} \cap N^{--}=\emptyset$ then $\left(N ; \cdot, e, N^{+}, N^{-}\right)$is indeed a \pm -semigroup, and by construction, (M5) holds.

Now assume we have a finite structure $\left(N ; \cdot, e, N^{++}, N^{--}, \leqslant\right)$, where $N^{++}$and $N^{--}$are defined as above, but with $N^{++} \cap N^{--} \neq \emptyset$. Can we associate to this an algebra whose D-reduct has Priestley dual $N$, and whose operations are determined dually by the left semigroup action on $N$ ? The problem is to decide how we should associate operations to the points $\mu \in N^{++} \cap N^{--}$. Naively, we might try to overcome 
this difficulty simply by requiring $f_{\mu}$ for $\mu \in N^{++} \cap N^{--}$to be both an endomorphism and a dual endomorphism. However, as already noted, this is impossible in a nontrivial algebra. We might try instead to select disjoint sets $N^{+}$and $N^{-}$such that

$$
N=N^{+} \cup N^{-}, \quad N^{+} \subseteq N^{++}, \quad N^{-} \subseteq N^{--},
$$

to make $\left(N ; \cdot, e, N^{+}, N^{-}, \leqslant\right)$a member of $\mathscr{M}$. Suppose, to take an extreme case, that $N$ is discretely ordered (equivalently, $K(N)$ is a Boolean lattice). Then $N^{++}=$ $N^{--}=N$, so that the sets $N^{++}$and $N^{--}$, far from telling us exactly how to assign elements of $N$ to $N^{+}$and $N^{-}$, give us no indication at all how this should be done. Certainly we could choose $N^{+}=N, N^{-}=\emptyset$ and get a \pm -semigroup (in Cornish's terminology ([12, 5.1]) we would say $\mathbf{N}$ is improper). However this may not lead to D-N being a variety we wish to study. This is exactly the situation arising in the $\mathbf{P}_{m, n}$ example below, where it is knowledge of the variety that tells us to seek to place the generator $g$ of $N$ into $N^{-}$, and to assign the remaining elements as dictated by (M3). We shall see that this sometimes leads to an assignment of points to $N^{+}$and $N^{-}$which is incompatible with the semigroup multiplication.

2.5. Example. The Ockham varieties $\mathbf{P}_{m . n}$. The defining identities within $\mathbf{O}$ for the variety $\mathbf{P}_{m, n}$ are:

$$
\begin{array}{ll}
\text { for } m-n \text { even: } & \sim^{m} a=\sim^{n} a, \\
\text { for } m-n \text { odd: } & \begin{cases}\sim^{m} a \wedge \sim^{n} a=0, \\
\sim^{m} a \vee \sim^{n} a=1 .\end{cases}
\end{array}
$$

For $m=2$ and $n=0$ we have the variety $\mathbf{M}=\mathbf{P}_{2,0}$ of de Morgan algebras. Theorem 2.3 provides the well-known dual category $\mathscr{Y}^{\mathbf{P}_{m . n}}$ : it is the full subcategory of $\mathscr{Y}^{\mathbf{0}}$ consisting of spaces $(Y ; g)$ on which $g^{m}=g^{n}$.

As is well known, $\mathbf{P}_{m, n}=\mathbb{U S P}(\underline{P})=\mathbb{H} \mathbb{S}(\underline{P})$ where $H(P)$ is an $m$-element antichain. This may be considered as the discretely ordered monoid

$$
N_{m, n}:=\left\{1, g, \ldots, g^{m-1}\right\} \quad \text { with } g^{m}=g^{n} .
$$

Here the cases $m-n$ even and $m-n$ odd diverge. Consider $m-n$ even. Take

$$
N_{m, n}^{+}=\left\{g^{k} \in N_{m, n} \mid k \text { even }\right\} \quad \text { and } \quad N_{m, n}^{-}=\left\{g^{k} \in N_{m, n} \mid k \text { odd }\right\} .
$$

This makes $N_{m, n}$ into an ordered \pm -monoid $\mathbf{N}_{m, n}$, and $\mathbf{P}_{m, n}=\mathbf{D}-\mathbf{N}_{m, n}$.

Now assume that $m-n$ is odd, and write $N$ for $N_{m, n}$. Again we wish $g$ to correspond to a dual-homomorphic negation, so must assign $g$ to $N^{-}$. Then the rule $N^{\sigma} \cdot N^{\tau} \subseteq N^{\sigma \tau}$ forces us to take $g^{k} \in N^{-}$for $k$ odd and $g^{k} \in N^{+}$for $k$ even. Thus $g^{n}=g^{m}$ forces $g^{n}$ to lie both in $N^{+}$and in $N^{-}$. Thus the semigroup multiplication 


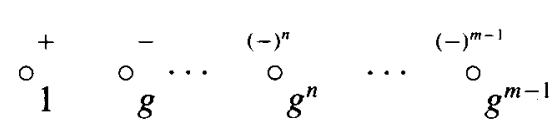

FIGURE 3. $\mathbf{N}^{\mathbf{P}_{m . n}}=\mathbf{N}_{m . n}(m-n$ even $)$

is incompatible with an assignment of the elements of $N$ to disjoint sets $\mathrm{N}^{+}$and $\mathrm{N}^{-}$ according to condition (M3) if we demand $g \in N^{-}$.

The preceding example shows that weak ordered \pm -semigroups cannot be used in the same simple way that ordered \pm -semigroups can. We now revert to generalities, and seek to broaden our definitions in order to fit this example and others like it into our semigroup framework. Let us look more closely at the example $\mathbf{P}_{m, n}$ ( $m-n$ odd). The clue on how to proceed is to be found in a simple observation about Ockham algebras, namely that $(2 m-n)-n$ is even and the inclusion $\mathbf{P}_{m, n} \subseteq \mathbf{P}_{(2 m-n), n}$ holds. Dually this comes from the fact that

$$
g^{m}=g^{n} \quad \text { implies } \quad g^{2 m-n}=g^{n+m-n}=g^{m}=g^{n}
$$

algebraically it is the statement that $\mathbf{P}_{m, n}$ lies in the Berman class $\mathbf{K}_{m-n, n}$. Define a map

$$
F: g^{k} \mapsto \begin{cases}g^{k} & \text { if } k<m, \\ g^{k-m+n} & \text { if } m \leqslant k<2 m-n,\end{cases}
$$

from $N_{(2 m-n) . n}$ onto $N_{m, n}$. Informally, we wind the $2(m-n)$-element loop of $N_{(2 m-n), n}$ twice round the $(m-n)$-element loop of $N_{m, n}$. The map $F$ is a monoid homomorphism. We may then define

$$
\begin{aligned}
& \left(N_{m, n}\right)^{+}:=F\left(\left(N_{(2 m-n), n}\right)^{+}\right)=\left\{g^{k} \mid k \text { is even or } n \leqslant k<m\right\} \\
& \left(N_{m . n}\right)^{-}:=F\left(\left(N_{(2 m-n) . n}\right)^{-}\right)=\left\{g^{k} \mid k \text { is odd or } n \leqslant k<m\right\} .
\end{aligned}
$$

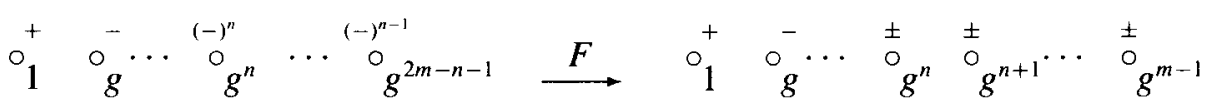

$$
\begin{aligned}
& N_{(2 m-n) . n} \quad N_{m, n}
\end{aligned}
$$

FIGURE 4

We may regard $\mathbf{N}:=\mathbf{N}_{m . n}$ as the image of $\mathbf{M}:=\mathbf{N}_{(2 m-n) . n}$ under the $\mathbf{M}-\mathbf{P}$ morphism $F$. Thus $\mathbf{N}$ is certainly the dual of an algebra $\underline{P}$ in $\mathbf{D}-\mathbf{M}$, which has operations $\left(f_{\mu}^{M}\right\}_{\mu \in M}$, 
say. It is clear that $F^{-1}(v)$ consists either of a single point (if $v=g^{k}$ for $k<n$ ) or of a pair of points, one in $\mathrm{M}^{+}$and one in $M^{-}$. If $\mu_{1}$ and $\mu_{2}$ are distinct points identified by $F$ then on $\underline{P}$ the associated operations $f_{\mu_{1}}^{M}$ and $f_{\mu_{2}}^{M}$ are linked by the identities corresponding to $g_{\mu_{1}}^{M}=g_{\mu_{2}}^{M}$ according to the rule given in Theorem 2.3. We thus have two algebraic operations linked to points $\mu \in N^{+} \cap N^{-}$, one an endomorphism and the other a dual endomorphism.

Thus in building an algebra from $\mathbf{N}$ we are led to associate a pair of operations to each point in $N^{+} \cap N^{-}$(one endomorphism and one dual endomorphism) and just one to each point in the symmetric difference $N^{+} \Delta N^{-}$. To formalise this process we proceed as follows. We take $\mathbf{N}=\left(N ; \cdot, e, N^{+}, N^{-}, \leqslant\right)$to be a finite weak ordered \pm -semigroup. Let $C$ denote the 2-element cyclic group with elements \pm 1 equipped with the discrete order. Define

$$
\bar{N}=\left(N^{+} \times\{1\}\right) \cup\left(N^{-} \times\{-1\}\right),
$$

with order, $\leqslant$, and multiplication, $\cdot$, inherited from $N \times C$. With this multiplication, $\bar{N}$ is a subsemigroup of $N \times C$, and by defining $\bar{N}^{+}=N^{+} \times\{1\}$ and $\bar{N}^{-}=$ $N^{-} \times\{-1\}$ we make $\bar{N}$ into a \pm -semigroup. Because $\mathbf{N}$ satisfies (M5) and the order on $C$ is discrete, $\overline{\mathbf{N}}$ satisfies (M5) under its product order, $\leqslant^{\prime}$. Thus $\overline{\mathbf{N}}=$ $\left(\bar{N} ; \cdot,(e, 1), \bar{N}^{+}, \bar{N}^{-}, \leqslant^{\prime}\right)$ is an ordered \pm -semigroup, which we say is canonically associated to $\mathbf{N}$. Moreover, the natural projection map $F$ from $\overline{\mathbf{N}}$ onto $\mathbf{N}$ is an order-preserving semigroup homomorphism with $F\left(\bar{N}^{\sigma}\right)=N^{\sigma}$ for $\sigma \in\{ \pm\}$.

Given $\mathbf{N} \in \mathscr{N}$ we define $g_{v}$ as, before to be left multiplication by $v$ and define an algebra $\underline{P}$ by taking the $\mathbf{D}$-reduct of $\underline{P}$ to be $K(N)$ and assigning operations $f_{\mu}$ for $\mu \in \bar{N}$ by

$$
\begin{aligned}
f_{(v, 1)}(a)(x) & =a\left(g_{v}(x)\right), \\
f_{(v,-1)}(a)(x) & =1-a\left(g_{\nu}(x)\right) .
\end{aligned}
$$

Define, on $N, \bar{g}_{\mu}=g_{v} \quad$ for $\mu \in F^{-1}(v)$ and, dually, on $K(N)$,

$$
\bar{f}_{\mu}=\left\{\begin{array}{llll}
f_{(v, 1)} & \text { if } \mu \in \bar{N}^{+} & \text {and } & F(\mu)=v, \\
f_{(v,-1)} & \text { if } \mu \in \bar{N}^{-} & \text {and } & F(\mu)=v .
\end{array}\right.
$$

These operations are well defined, and make $N$ into an object in $\overline{\mathbf{N}}-\mathbf{P}$ and $K(N)$ into an algebra dual to it, $\underline{P}$ in $\mathbf{D}-\overline{\mathrm{N}}$. Furthermore, $\underline{P}$ is term-equivalent to $\underline{P}^{\overline{\mathrm{N}}}$.

Because the operations $\left\{\bar{g}_{\mu}\right\}_{\mu \in \bar{N}}$ act on $N$ as $\bar{g}_{\mu}=g_{\nu}$ for $\mu \in F^{-1}(\nu)$, any two operations $\bar{f}_{\mu_{1}}$ and $\bar{f}_{\mu_{2}}$ coincide on $\underline{P}^{\bar{N}}$ precisely when $\mu_{1}=\mu_{2}$ or $\mu_{1}=(\nu, \varepsilon)$ and $\mu_{2}=(\nu, 1-\varepsilon)$, where $v \in N$ and $\varepsilon \in C$. The equality of $\bar{g}_{\mu_{1}}$ and $\bar{g}_{\mu_{2}}$ leads to a pair of identities linking $\bar{f}_{\mu_{1}}$ and $\bar{f}_{\mu_{2}}$, according to Theorem 2.3. We have shown that we may associate an algebra $\underline{P}^{\mathrm{N}}$ to $\mathrm{N}$ by assigning an operation $f_{\mu}$ to each $\mu$ in 
$\bar{N}$. Otherwise stated, we associate with each $v \in N^{+} \cap N^{-}$a pair of operations, one an endomorphism and the other a dual endomorphism, one linked to $g_{v} q u a$ orderpreserving map and one linked to $g_{\nu} q u a$ order-reversing map. The algebra $\underline{P}^{\mathrm{N}}$ is the one we shall henceforth associate to a given weak ordered \pm -semigroup $\mathbf{N}$. This is not at variance with our usage for $\mathbf{N} \in \mathscr{M}$, since then $\mathbf{N} \cong \overline{\mathbf{N}}$, as ordered \pm -semigroups. We extend to the wider class $\mathscr{N}$ the notations $\mathbf{N}$ for $N$ thought of as a dual space and $\mathbf{N}^{\mathscr{A}}$ for a semigroup which acts, as in our discussion above, as the dual space of an algebra generating a variety $\mathscr{A}$. Further, we define $\mathbf{D}-\mathbf{N}:=\mathbf{D}-\overline{\mathbf{N}}$, and let $\mathbf{N}-\mathbf{P}$ be the corresponding dual category of Priestley spaces with a continuous left $N$-action. Again this does not conflict with earlier usage.

Since it is so central to our approach we sum up our preceding discussion in the following theorem, streamlining the notation a little.

THEOREM 2.6. Let $\mathbf{N}$ be a finite weak ordered \pm -semigroup, with canonically associated ordered \pm -semigroup $\overline{\mathbf{N}}$. Then there exists a finite algebra

$$
\underline{P}^{\mathrm{N}}=\left(K(N) ; \wedge, \vee, 0,1,\left\{f_{\mu}^{+}\right\}_{\mu \in N^{+}},\left\{f_{\mu}^{-}\right\}_{\mu \in N^{-}}\right)
$$

in D-N such that $H\left(P^{\mathbf{N}}\right)=\mathbf{N}$ and

(i) for all $a \in \underline{P}^{\mathrm{N}}$ and all $v \in N$,

$$
\begin{array}{ll}
f_{\mu}^{+}(a)(\nu)=a(\mu \nu) & \text { for all } \mu \in N^{+}, \\
f_{\mu}^{-}(a)(\nu)=1-a(\mu \nu) & \text { for all } \mu \in N^{-}
\end{array}
$$

(ii) $\underline{P}^{\mathrm{N}}$ satisfies the identities

$$
f_{\mu}^{+}(a) \wedge f_{\mu}^{-}(a)=0 \text { and } f_{\mu}^{+}(a) \vee f_{\mu}^{-}(a)=1,
$$

whenever $\mu \in N^{+} \cap N^{-}$.

Different weak ordered \pm -semigroups may give rise to the same canonically associated ordered \pm -semigroup. The disjointification of $N^{+}$and $N^{-}$serves only to allow us to define the algebraic operations on the algebra dual to a weak ordered \pm -semigroup. It is the order of the original weak semigroup which determines the identities that these operations are subject to.

The next clutch of examples is of varieties easily recognised to be of the type $\mathscr{A}^{\mathrm{N}}$ for $\mathbf{N} \in \mathscr{N}$ but which are not of the type $\mathscr{A}^{\mathbf{M}}$ for $\mathbf{M} \in \mathscr{M}$. The first example simply records the conclusion of our discussion above.

2.7. Examples. (1) $\mathbf{P}_{m, n}\left(m-n\right.$ odd). Write $\mathbf{N}$ for the weak ordered monoid $\mathbf{N}_{m, n}$. Then the ordered monoid $\overline{\mathbf{N}}$ is isomorphic to $\mathbf{N}_{(2 m-n), n}$. 
(2) Stone algebras. The variety $\mathbf{S}$ of Stone algebras is $\mathbf{D}-\mathbf{N}^{\mathbf{S}}$ where $\mathbf{N}^{\mathbf{S}}$ is as shown in Figure 5(a). The canonically associated \pm -monoid $\overline{\mathbf{N}}^{\mathbf{S}}$ is $\mathbf{N}^{\mathrm{MS}}$.

(3) Double Stone algebras. The variety of double Stone algebras is $\mathbf{D}-\mathbf{N}^{\mathbf{D S}}$, where $\mathbf{N}^{\text {DS }}$ is as shown in Figure 5. The corresponding monoid $\overline{\mathbf{N}}^{\mathbf{D S}}$ is $\mathbf{N}^{\text {DMS }}$.

$$
\begin{aligned}
& \pm \\
& +\int_{1}
\end{aligned}
$$

(a) $\mathbf{N}^{\mathrm{S}}$

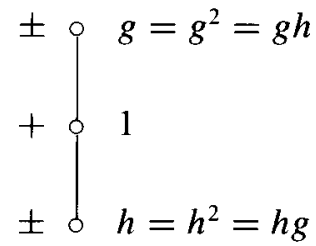

(b) $\mathbf{N}^{\mathrm{DS}}$

FIGURE 5

(4) Linked weak double Stone algebras. This class of algebras was studied by Sankapannavar in [34]. It forms a variety LWDS, derived from the same weak \pm -monoid as in (3), but with the discrete order.

Various distributive-lattice-ordered algebras inspired by non-classical propositional logics, and related to $n$-valued Łukasiewicz algebras, have been explored by Cignoli and Gallego in [9]. The common feature of all these is a de Morgan negation linked to an operation which preserves either $\vee$ or $\wedge$. Two classes of this type-involutive Stone algebras and regular $\alpha$-de Morgan algebras-are shown to be finitely generated varieties which can be cast in the form $\mathscr{A}^{\mathrm{N}}$.

2.8. Examples. (1) Regular $\alpha$-de Morgan algebras. In [9], the variety $\mathbf{R}$ of regular $\alpha$-de Morgan algebras is defined equationally in terms of a de Morgan negation $\sim$ and an operation $\alpha$, and shown to be generated by an algebra with the 5-element Kleene chain $0<a<b=\sim b<\sim a=1$ as a reduct. On this chain, $\alpha$ acts as a D-endomorphism sending $a$ and $b$ to 0 and $\sim a$ to 1 . From this we see easily that $\mathbf{R}=\mathscr{A}^{\mathbf{N}}$, where $\mathbf{N}=\mathbf{N}^{\mathbf{R}}$ is as shown in Figure 6.

.(2) Involutive Stone algebras. The variety of involutive Stone algebras can be identified with $\mathbf{D}$-N where the monoid $N$ is

$$
N=\{1, n, g, g n\} \quad \text { with } n^{2}=n, g^{2}=1, n g=n,
$$

and the order and \pm assignment are as shown in Figure 7(a). The canonically associated ordered \pm -monoid is as shown in Figure 7(b). 


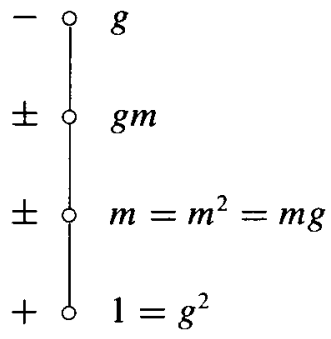

FIGURE 6. $\mathbf{N}^{\mathbf{R}}$

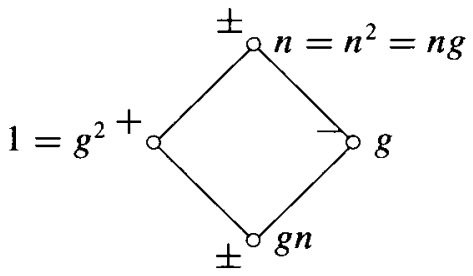

(a) $\mathbf{N}^{I S}$

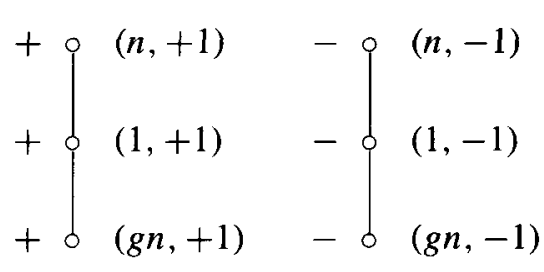

(b) $\overline{\mathbf{N}}^{\mathbf{I S}}$

FIGURE 7

(3) Kleene-Stone algebras. MS-algebras provide a common generalisation of de Morgan and Stone algebras. A different umbrella variety for the varieties $\mathbf{K}$ and $\mathbf{S}$ has been studied by Guzman and Squier [23] under the name Kleene-Stone algebras. It is shown in [23] that the subdirectly irreducible algebras in this variety, KS, are the subalgebras of the 5-element chain equipped with (necessarily unique) unary operations under which it is both a Kleene algebra and a Stone algebra. Therefore $\mathbf{K S}=\mathbb{\square} \mathbb{P}(K(\mathbf{N}))$ where $\mathbf{N} \in \mathscr{N}$ is as shown in Figure 8(a).

Comparing the monoid diagrams for IS and KS we recognise KS as a subvariety of IS: the space $\mathbf{N}^{\mathbf{K S}}$ is in $\mathbf{N}^{\mathbf{I S}}$-P. Observe that the ordered monoid canonically associated to $\mathbf{N}^{\mathrm{KS}}$ is the same as that associated to $\mathbf{N}^{\mathbf{I S}}$.

(4) 3-valued Lukasiewicz algebras. Consider the monoid $\mathbf{N}$ shown in Figure $8(\mathrm{~b})$. Notice that we have here a semigroup which fails to be a monoid. It is the dual space of a double Stone algebra and the variety generated by $\underline{P}^{\mathrm{N}}$ is a subvariety $\mathscr{A}$ of DS. It is well known that the members of the class $\mathscr{A}$ are exactly the 3-valued Łukasiewicz algebras: the class $\mathscr{A}$ can be characterised within DS as the semi-simple algebras (see [9, Theorem 2.9], or [4, Theorem 5.1]). Alternatively, the 


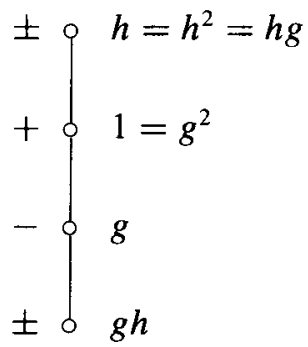

(a) $\mathbf{N}^{\mathrm{KS}}$

$$
\begin{array}{ll} 
\pm \quad h=h^{2}=h g \\
\pm \delta \quad g=g^{2}=g h
\end{array}
$$

(b) $\mathbf{N}^{\mathbf{L}_{3}}$

FIGURE 8

Kleene negation can be captured through conditions on the complemented elements (see [8], where attention is drawn to the import of the results on p. 220 of [2]).

Now consider the class $\mathbf{L}_{n}$, of $n$-valued Łukasiewicz algebras without negation, for $n>3$. When $n=3$ we have seen that the Kleene negation can be captured in a fortuitous manner when it is not included in the type. In the cases $n=4,5$ it is shown in [9], Section 4, that the class $\mathbf{L}_{n}$ consists of those algebras which have reducts lying in the subvarieties of IS and of $\mathbf{R}$ generated by an $n$-element chain. This already suggests that we may not be able to regard the variety $\mathbf{L}_{n}$, even for $n=4,5$, as being of the form $\mathscr{A}^{\mathbf{N}}$, where $\mathbf{N} \in \mathscr{N}$.

Cornish presents in $[12,5.4 .11]$, a \pm -monoid associated with $\mathbf{L}_{n}$ (and gives a similar discussion for the variety $\mathbf{M}_{n}$ of $n$-valued Łukasiewicz algebras without negation). The monoid $M$ for $\mathbf{L}_{n}$ has $2 n$ elements $1, \sigma_{1}, \ldots, \sigma_{n-1}, \sigma, \sigma \sigma_{1}, \ldots, \sigma \sigma_{n-1}$, with $\sigma^{2}=1$, $\sigma_{i} \sigma=\sigma \sigma_{n-i}$ and $\sigma_{i} \sigma_{j}=\sigma_{i}$, for $i, j \leqslant n-1$. The first $n$ elements belong to $M^{+}$, the remainder to $M^{-}$. The variety $L_{n}$ is generated by the $n$-element chain, whose dual is the $(n-1)$-element chain $\sigma_{1}<\sigma_{2}<\cdots<\sigma_{n-1}$, with the dual operations given by the monoid multiplication. This makes the set $\sigma_{1}, \ldots, \sigma_{n-1}$ into an element $\mathbf{N}$ in $\mathscr{N}$. However we have no element to assign to $\sigma$. We cannot circumvent the difficulty by working with $\mathbf{M}_{n}$ instead, and giving every point a \pm -label, since this does not give the right algebraic identities. We are forced to concede that we must think of $\sigma$ as an additional dual operation on $\mathbf{N}$. Formally this can be done by taking the $\mathbf{N}^{\mathrm{K}}$-expansions of the categories $\mathbf{N}-\mathbf{P}$ and $\mathbf{D}-\mathbf{N}$. We do not set up the associated duality theory for structures of this sort, since varieties like $\mathbf{L}_{n}$ are relatively rare, and can be treated directly by the piggyback duality method. We have studied in detail in [28] natural dualities for the varieties $\mathbf{L}_{n}$ and $\mathbf{M}_{n}$ of $n$-valued Łukasiewicz algebras with and without negation.

It is natural to ask whether every subvariety generated by a subdirectly irreducible 
algebra in a variety $\mathscr{A}^{\mathbf{N}}$ is itself of the same type. We shall show below that this is not the case, after we have discussed subdirectly irreducible algebras.

\section{Properties of the variety $\mathscr{A}^{\mathrm{N}}$ for $\mathbf{N} \in \mathscr{N}$}

The following lemma will be used both to reveal when the subdirectly irreducible algebras in a variety $\mathscr{A}^{\mathrm{N}}$ generated by a finite subdirectly irreducible algebra $\underline{P}^{\mathrm{N}}$ are always subalgebras of $\underline{P}^{\mathrm{N}}$, and also to tell us which relations we need to include in a natural duality for $\mathscr{A}^{\mathrm{N}}$. We shall fix $\mathbf{N} \in \mathscr{N}$, and write $\underline{P}$ in place of $\underline{P}^{\mathrm{N}}$ and $\mathscr{A}$ in place of $\mathscr{A}^{\mathrm{N}}$. The Priestley dual category for $\mathscr{A}$ is denoted $\mathscr{Y}^{\mathscr{A}}$, as usual.

LEMMA 3.1. Let $\mathbf{N} \in \mathscr{N}$. For each $\mu \in N$, the map $\eta_{\mu}: \nu \mapsto v \mu$ is the dual of an endomorphism $u_{\mu}: \underline{P} \rightarrow \underline{P}$ if and only if it is order-preserving. In this case the dual map $K\left(\eta_{\mu}\right)$ is given by $u_{\mu}(a)(v)=a(v \mu)$. Furthermore,

$$
\text { End } \underline{P}=\left\{K\left(\eta_{\mu}\right) \mid \eta_{\mu} \text { is order-preserving }\right\} \text {. }
$$

PROOF. For any $\lambda, \mu, \nu \in N$,

$$
g_{\lambda}\left(\eta_{\mu}(\nu)\right)=\lambda(\nu \mu)=(\lambda \nu) \mu=\eta_{\mu}(\lambda \nu)=\eta_{\mu}\left(g_{\lambda}(\nu)\right) .
$$

Thus $\eta_{\mu}$ is an $\mathbf{N}$-P-morphism so long as it is order-preserving.

Write $u_{\mu}:=K\left(\eta_{\mu}\right)$. We have, for all $a \in \underline{P}$ and $v \in N$,

$$
u_{\mu}(a)(v)=a\left(\eta_{\mu}(v)\right)=a(v \mu) .
$$

For the final statement, note that if $\varphi$ is dual to an endomorphism of $\underline{P}$ then

$$
\varphi(v)=\varphi(v e)=\varphi\left(g_{v}(e)\right)=g_{v}(\varphi(e))=v(\varphi(e)),
$$

so that $\varphi=\eta_{\varphi(e)}$.

Note the way in which on $\mathbf{N}$ we obtain duals of endomorphisms by right multiplication (under restricted conditions) and of operations by left multiplication.

Our next task is to extend to our setting some results on congruences and subdirectly irreducible algebras proved in the \pm -monoid setting by Cornish, subsuming results obtained earlier by those who led the way by studying special cases.

Proposition 3.2. (see [12,9.6.3]) Let $\mathbf{N} \in \mathscr{N}$ and let $A \in \mathbf{D}-\mathbf{N}$. There is a lattice anti-isomorphism between the lattice of closed $N$-closed subsets of $H(A)$ and the lattice of congruences of A given by $T \mapsto \Theta_{T}$, where

$$
a \equiv b\left(\Theta_{T}\right) \text { if and only if } a \uparrow_{T}=b \uparrow_{T}
$$

for all $a, b$ in $A$. (Here a subset of a space in N-P is $N$-closed if it is closed under the action of the maps $g_{\nu}(v \in N)$ on that space.) 
PROOF. Under Priestley duality, D-congruences correspond to topologically closed subsets $Y$ of $H(A)$ under the mapping $\Theta_{T} \leftrightarrow T$, and $\Theta_{T}$ is an D-N-congruence if and only if $Y$ is $N$-closed.

Proposition 3.3. Let $\mathbf{N} \in \mathscr{N}$. Then any subclass of $\mathbf{D}-\mathbf{N}$ has the Congruence Extension Property.

PROOF. This is proved exactly as in $[12,8.21 .4]$.

Mimicking the notation adopted by Goldberg [22] in the Ockham setting, we shall write $\mathbb{G}$ and $\mathbb{M}$ for the operators dual to $\mathbb{N}$ and $\mathbb{S}$. (CEP) implies that (up to isomorphisms) $\mathbb{H}$ and $\mathbb{S}$ commute, so $\mathbb{G}$ and $\mathbb{M}$ do too.

The next result is immediate from Proposition 3.2.

Proposition 3.4. ([12, 9.6.5]) Let $\mathbf{N} \in \mathscr{N}$. Then a finite algebra $A \in \mathbf{D}-\mathbf{N}$ is subdirectly irreducible if and only if there exists $z \in H(A)$ such that

$$
H(A)=\left\{g_{\mu}(z) \mid \mu \in N\right\} .
$$

In particular, the algebra $\underline{P}^{\mathbf{N}}$ is subdirectly irreducible, with e serving as $z$.

For any $z \in Y, Y \in \mathbf{N}$-P; we shall henceforth write $\left\{g_{\mu}(z) \mid \mu \in N\right\}$ as $N z$.

We now invoke the standard machinery of universal algebra (see, for example, [7]). Any variety $\mathscr{A}$ generated by a finite algebra $\underline{P}$ with a distributive lattice reduct is congruence-distributive. By Jónsson's Lemma, the set $\mathrm{Si}(\mathscr{A})$ of (isomorphism classes of) subdirectly irreducible algebras in $\mathscr{A}$ is a subset of the set $\mathbb{O N S}(\underline{P})$ of homomorphic images of subalgebras of $\underline{P}$. Hence by Birkhoff's Subdirect Product Theorem and Birkhoff's $\mathbb{H S P}$ Theorem,

$$
\mathscr{A}=\mathbb{U S P}(\operatorname{Si}(\mathscr{A}))=\mathbb{U S P}(\mathbb{H S}(\underline{P})) .
$$

Furthermore, given that (CEP) holds, the right-hand expression simplifies to give us $\mathscr{A}=\mathbb{V S P}(\mathbb{H}(P))$. In the case that every homomorphic image of $\underline{P}$ is a subalgebra of $\underline{P}$, then we have $\mathscr{A}=\mathbb{U S P}(\underline{P})$. More precisely, $\mathbb{U S P}(\underline{P}) \varsubsetneqq \mathscr{A}$ if and only if $(\mathbb{O}(P) \backslash \mathbb{Q}(P)) \cap \operatorname{Si}(\mathscr{A}) \neq \emptyset$.

The next result extends [12, Theorem 9.6.6]; see also [22, Corollary 2.9].

THEOREM 3.5. Let $\mathbf{N} \in \mathscr{N}$. Then the following hold.

(i) $\underline{P} \in \operatorname{Si}(\mathscr{A})$.

(ii) $\operatorname{Si}(\mathscr{A}) \subseteq \mathrm{HS}(P)$.

(iii) $\mathbb{S}(P) \subseteq \operatorname{Si}(\mathscr{A})$. 
(iv) The subdirectly irreducible algebras in $\mathscr{A}$ are precisely the homomorphic images of subalgebras of $\underline{P}$ if and only if every left ideal of the semigroup $N$ is principal.

(v) The subdirectly irreducible algebras in $\mathscr{A}$ are precisely the (isomorphic copies of) subalgebras of $\underline{P}$ if and only if every principal left ideal of $N$ is expressible in the form $N \mu$ with the map $\nu \mapsto \nu \mu$ order-preserving. This happens in particular if $N$ is discretely ordered.

PROOF. Statements (i) and (ii) have already been noted.

Consider (iii). Let $\underline{Q}$ be a subalgebra of $\underline{P}$. We have that $H(\underline{Q})$ is the image of $\mathbf{N}$ under a $\mathscr{Y}^{\mathscr{A}}$-morphism $\varphi$. Then $H(\underline{Q})=\{\nu \varphi(e) \mid \nu \in N\}$, whence $\underline{Q}$ is subdirectly irreducible.

For (iv), suppose that every left ideal in $N$ is principal. Let $\underline{Q}$ be a homomorphic image of a subalgebra of $\underline{P}$, so $H(Q)$ is an $N$-closed subset of an image under a $\mathscr{Y}^{\mathscr{A}}$-morphism $\varphi$ with domain $N$. Define

$$
J:=\{\mu \in N \mid \varphi(\mu) \in H(Q)\}
$$

Then $J$ is a left ideal of $N$, so of the form $N v$ for some $v \in N$. But then $H(Q)=$ $N \varphi(v)$, so $\underline{Q}$ is subdirectly irreducible. Conversely, let every homomorphic image of a subalgebra of $\underline{P}$ be subdirectly irreducible. This means in particular that every $N$-closed subset of $N$ is of the form $N \mu$, that is, every left ideal of $N$ is principal.

For (v), note that any homomorphic image $\underline{Q}$ of $\underline{P}$ has $H(Q)$ identifiable with some $N$-closed subset of $N$, and that $\underline{Q}$ is subdirectly irreducible if and only if this subset is a principal left ideal. We now invoke Lemma 3.1.

The following corollary is in the same spirit as [16, Theorem 3.15], which yields the result $[17,3.2]$ for Ockham algebras which the corollary generalises.

Corollary 3.6. Let $\mathbf{N} \in \mathscr{N}$ and let

$$
\mathscr{J}:=\left\{N \mu \mid \mu=e \text { or } \neg\left((\exists v) N \nu=N \mu \text { and } \eta_{v} \text { is order-preserving }\right)\right\} .
$$

Order $\mathscr{J}$ by $J_{1} \ll J_{2}$ in $\mathscr{J}$ if and only if there exist $\nu_{i} \in N$ such that $J_{i}=N \nu_{i}$ $(i=1,2)$ such that $v v_{2} \mapsto v v_{1}$ is an order-preserving map from $J_{2}$ onto $J_{1}$. Then

$$
\mathbb{M S P}(\underline{P})=\mathbb{U S P}(\underline{\Pi}) \text { where } \underline{\Pi}=\{K(J) \mid J \text { is maximal in } \mathscr{J}\} \text {. }
$$

PROOF. We have $\mathbb{H} \ \mathbb{P}(\underline{P})=\square \mathbb{S}(\mathbb{H}(\underline{P}))$. If the restriction to maximal elements were removed the stated result would be immediate from Lemma 3.1. The order is defined so that $J_{1} \ll J_{2}$ if and only if $K\left(J_{1}\right)$ is isomorphic to a subalgebra of $K\left(J_{2}\right)$. Therefore only maximal elements of $\mathscr{J}$ need to be included in $\underline{\Pi}$. 
3.7. Examples. (1) Double MS-algebras. Consider DMS, with $\mathbf{N}=\mathbf{N}^{\mathrm{DMS}}$ as in 2.4(2). In this case the proper principal left ideals are

$$
N g=N g^{2}=N h=N h^{2}=N \backslash\{1\} .
$$

We claim that the maps $v \mapsto \nu g$ and $\nu \mapsto v h$ are order-preserving. We have

$$
\begin{array}{rll}
h^{2} \leqslant 1 \leqslant g^{2} & \text { implies } & h^{2} g=h \leqslant g=g^{2} g, \\
h \leqslant g & \text { implies } & h g=h^{2} \leqslant g^{2}=g g .
\end{array}
$$

Thus $\eta_{g}$ is order-preserving, and a similar argument shows that $\eta_{h}$ is also orderpreserving. It follows that the map $\eta_{\nu}$ is order-preserving for every $v \in N$, so that the subdirectly irreducible algebras in DMS are precisely the subalgebras of the generating algebra (a result previously proved algebraically (see [5] or [6, Ch. 13]), and we have that the variety DMS is $\mathbb{D S P}(\underline{P})$, where $H(\underline{P})=\mathbf{N}^{\mathrm{DMS}}$.

(2) Ockham varieties. It was pointed out in [17] that every Ockham variety generated by a finite subdirectly irreducible algebra is $\mathbb{U S P}(\underline{P}, \underline{Q})$, where $H(\underline{Q})=$ $g(H(\underline{P}))$. Note that the monoid is commutative, so the left and right monoid actions by powers of $g$ coincide. Necessary and sufficient conditions are given in [17, Theorem 3.10], for $\mathbb{S P}(\underline{P})$ to coincide with $\mathbb{S} \mathbb{P}(\underline{P}, \underline{Q})$. These conditions are those that guarantee that either $g: N \rightarrow N g$ is order-preserving or $g^{2}$ maps $N$ onto $N g$. The latter are exactly the ways in which we can have $N g \ll N$ in the order of Corollary 3.6. As [17, Theorem 3.10] indicates, the conditions for these to be satisfied are various, involving the interaction between the order and the monoid action.

(3) Kleene-Stone algebras. Let $\mathbf{N}:=\mathbf{N}^{\mathrm{KS}}$. We have one proper left ideal, namely the principal ideal $N h=N g h=\{g h, h g\}$. The maps $\eta_{1}(=\mathrm{id})$ and $\eta_{h}$ (which identifies the pairs $1, g$ and $g, g h$ ) are order-preserving, while $\eta_{g}$ and $\eta_{g h}$ are not. Accordingly, the subdirectly irreducible algebras in $\mathbf{K S}$ are exactly the subalgebras of $\underline{P}$ in this case (as shown in [23]), and the variety and quasivariety generated by this algebra coincide.

It is easy to exhibit an example in which $\mathscr{A}^{\mathbf{N}}$ is such that not every homomorphic image of $\underline{P}$ is subdirectly irreducible. We may simply take $N$ to be the monoid with two generators $\alpha$ and $\beta$ for which $\alpha^{2}=\beta \alpha=\alpha$ and $\beta^{2}=\alpha \beta=\beta$, and make this into an ordered \pm -monoid by taking the discrete order and $N^{+}=N$. The subset $\{\alpha, \beta\}$ is a left ideal which is not principal.

By moving from Cornish's discretely ordered \pm -monoids to our larger class $\mathscr{N}$, we have extended the class of varieties which can be treated intrinsically. As a consequence of Jonsson's Lemma, the set of join-irreducible elements in the (finite distributive) lattice of subvarieties of $\mathscr{A}:=\mathscr{A}^{\mathrm{N}}$ is order-isomorphic to $\operatorname{Si}(\mathscr{A})$, as described in [14]. We may ask whether our class $\mathscr{N}$ is closed under the operators 
$\mathbb{G}$ and $\mathbb{M}$. Consider a proper principal ideal $J=N z$ of $N$, where $\mathbf{N} \in \mathscr{N}$. Then $J$, with the inherited structure, belongs to $\mathscr{N}$ if and only if $J$ has a right identity, $e_{J}$. Certainly $e_{J}$ cannot be the original right identity $e$ of $N$, since $J$ is proper. Writing $e_{J}$ as $\mu z$ we see that we must have $(\nu z)(\mu z)=\nu z$ for all $\nu$, which is a rather restrictive condition; in particular we would need $(\mu z)^{2}=\mu z$. For a simple example of failure, take $\mathbf{N}=\mathbf{N}^{\mathbf{P}_{4.3}}$ and $J=N \backslash\{1\}$. Then the 3-element loop $J$ is generated by each of its elements, but none of these is idempotent. Hence $\mathscr{N}$ is not closed under $\mathbb{G}$.

Now suppose $\mathbf{N}^{\prime} \in \mathbb{M}(\mathbf{N})$. If $\mathbf{N}^{\prime}$ is obtained from $\mathbf{N} \in \mathscr{N}$ simply by replacing the order $\leqslant$ of $\mathbf{N}$ by a stronger order $\leqslant$ in such a way that the identity map is an $\mathbf{N}$-P-morphism, then $\mathbf{N}^{\prime}$ certainly belongs to $\mathscr{N}$. In general $\mathbf{N}$-P-morphic images of $\mathbf{N}$ do not have the structure of objects in $\mathscr{N}$. To illustrate, consider the variety DMS. In this case, the finite subdirectly irreducible algebras are exactly the subalgebras of the generating algebra $\underline{P}$. Consider the subdirectly irreducible algebra whose dual is given by collapsing the points $g$ and $h$ in $\mathbf{N}^{\text {DMS }}$. Denote the map effecting the collapse by $\psi$; see Figure 9. This algebra, $Q$, is that denoted $\operatorname{SID}_{19}$ in [6]. Suppose we try to make this into a semigroup $N^{\prime}$, so that the left semigroup action gives the maps dual to the DMS operations. The right identity element $e_{N}$ is forced to be $\psi(1)$, since it fails to be an image under any of the operations $g, h, g^{2}, h^{2}$. Denote the common image of $g$ and $h$ under $\psi$ by $\nu$. We must have the $g$-map given by left multiplication by some element of the semigroup $N^{\prime}$, and this element must be $v$, so that $\nu^{2}$ would have to be $v^{2}=\psi\left(h^{2}\right)$, so we would have a contradiction. So $\mathscr{N}$ is not closed under $\mathbb{M}$.

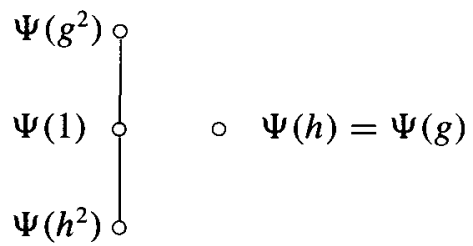

FIGURE 9

The above discussion shows that we must continue to regard certain varieties merely as subvarieties of varieties of type $\mathscr{A}^{\mathrm{N}}$, and to obtain natural dualities for them by a restriction process. It could therefore be argued that we have gained little by working intrinsically with any varieties outside Cornish's class of \pm -monoids. We contend that our focus on $\mathscr{N}$ is justified because it allows the direct analysis of many varieties important in their own right, and because the natural dualities we obtain for these varieties are so closely tied to the structure of the associated ordered semigroups. Arbitrary subvarieties of such varieties, by contrast, are often best analysed as subvarieties. As we show in [31], this allows us to determine 
natural dualities simultaneously for all subvarieties, and easily to address questions on equational bases.

Before leaving the topic of subdirectly irreducible algebras we make a final remark on non-uniqueness, attributable to the semigroup structure, not the order. The generating element $z$ in a principal ideal $J$ is generally not uniquely determined. In the Ockham case this occurs precisely when $J$ is a loop, when, in the terminology of [22], every element is an end. It happens also, for example, for $J=N \backslash\{1\}$ when $\mathbf{N}=\mathbf{N}^{\mathbf{P}_{3,1}}$; see 2.5. This phenomenon is more a nuisance than a serious impediment: it can make the statements of results more involved than they would otherwise be, and adds extra code to computer programs for determining the ordering $(\underline{Q} \leq \underline{R}$ if and only if $\underline{Q} \in \mathbb{H S}(\underline{R})$ ) on $\operatorname{Si}(\mathscr{A})$ used to analyse the lattice of subvarieties of $\mathscr{A}$ [14].

In [22, Theorem 2.11], Goldberg presented a description of the category $\mathscr{Y}^{\mathscr{A}}$ dual to a variety $\mathscr{A}$ generated by a finite subdirectly irreducible Ockham algebra $\underline{P}$. This showed that the spaces in $\mathscr{Y}^{\mathscr{A}}$ 'mimicked' the spaces $H(\underline{Q})$ for $\underline{Q} \in \mathbb{H} S(\underline{P})$ : the orbit under the $g$-map of a point in $Y \in \mathscr{Y}^{\mathscr{A}}$ satisfies the same inequalities as does the orbit of some end $e$ of some $H(Q)$. This theorem was refined and simplified in [16, Theorem 3.15], (and used in this simplified form to derive the natural dualities given in [17]). It was shown that it sufficed to consider inequalities satisfied within the $g$-orbits of just two points of $H(\underline{P})$, namely $e$ and $g(e)$, where $e$ is some (arbitrarily chosen) fixed end of $H(P)$. This comes about through the arguments given in connection with Proposition 3.6. For the general case we are content with the brute force result below, involving the inequalities in the orbits of all points of $H(\underline{P})$. The proof is essentially the same as that for Goldberg's original theorem.

Until further notice we fix $\mathbf{N} \in \mathscr{N}$ and $\mathscr{A}:=\mathscr{A}^{\mathrm{N}}$. We need some notation, following $[22,17]$. Let $B$ be a finite algebra (not necessarily subdirectly irreducible) in $\mathscr{A}$ and denote $H(B)$ by $W$. For $y \in Y, Y \in \mathscr{Y}^{\mathscr{A}}$, let

$$
\sigma^{W}(y):=\bigvee\left\{\sigma_{w}(y) \mid w \in W\right\}
$$

where

$$
\sigma_{w}(y):=\bigwedge_{\mu, v \in N}\left\{g_{\mu}(y) \leqslant g_{v}(y) \mid g_{\mu}(w) \leqslant g_{v}(w) \text { in } W\right\}
$$

Furthermore, let

$$
Y^{w}:=\left\{y \in Y \mid Y \models \sigma_{w}(y)\right\}
$$

so that $Y^{w}$ is the subspace of points which mimic the orbit $N w$ in $W$. (The set $Y^{w}$ is topologically closed since the operations $g_{\mu}$ and $g_{v}$ are continuous.) Finally, for any $A \in \mathscr{A}$, define $\Phi_{w}: \mathscr{A}(A, K(W)) \rightarrow H(A)$ by $\Phi_{w}(x):=w \circ x$.

We shall need the following result drawn from [17]. 
LEMMA 3.8. Let $\mathbf{N} \in \mathscr{N}$ and let $\mathscr{B}:=\mathbb{\triangle} \mathbb{P}\left(\underline{Q}_{1}, \ldots, \underline{Q}_{r}\right)$, where $\underline{Q}_{1}, \ldots, \underline{Q}_{r}$ are finite. Then, for $A \in \mathscr{B}$,

$$
H(A)=\bigcup\left\{\operatorname{im} \Phi_{w} \mid w \in W\right\}
$$

where $W:=H\left(\underline{Q}_{1}\right) \dot{\cup} \cdots \dot{\cup} H\left(\underline{Q}_{r}\right)$.

PROOF. Theorem 2.5 of [17] implies that every element of $H(A)$ is of the form $w_{i} \circ x_{i}$, where $x_{i} \in \mathscr{B}\left(A, \underline{Q}_{i}\right)$ and $w_{i} \in H\left(\underline{Q}_{i}\right)$. To get the result in the stated form, regard $w_{i}$ as a member of $\vec{W}_{\text {and }}$ compose $x_{i}$ with $\pi_{i}$, the canonical projection from $\underline{Q}_{1} \times \ldots \times \underline{Q}_{r}(\cong K(W))$ onto $\underline{Q}_{i}$.

THEOREM 3.9. Let $\mathbf{N} \in \mathscr{N}$. Let $\mathscr{B}=\mathbb{\Lambda} \mathbb{P}\left(\underline{Q}_{1}, \ldots, \underline{Q}_{r}\right)$ be a subvariety, where each $\underline{Q}_{i}$ is finite. Then

$$
\mathscr{B}=\{K(Y) \mid Y \in \mathbf{N}-\mathbf{P} \text { and } Y \models(\forall y) \sigma(y)\},
$$

where $\sigma(y):=\sigma^{W}(y), W=H\left(\underline{Q}_{1}\right) \dot{\cup} \cdots \dot{\cup} H\left(\underline{Q}_{r}\right)$.

Proof. Suppose $Y \in \mathbf{N}-\mathbf{P}$ is such that $Y \models(\forall y) \sigma(y)$. Fix $u \in Y$. Then $Y \models \sigma(u)$. Therefore there exists $w_{u} \in W$ such that $Y \models \sigma_{w_{u}}(u)$. Note that $N w_{u}$ is in $\mathbb{G}(W)$, so in $\mathscr{Y}^{\mathscr{B}}$. The map $\varphi_{u}: g_{\mu}\left(w_{u}\right) \mapsto g_{\mu}(u)$ is a well-defined $\mathbf{N}$-P-morphism from $N w_{u}$ into $Y$, because $\sigma_{w_{u}}(u)$ holds. Let $a \neq b \in K(Y)$ and assume without loss of generality that there exists $u \in Y$ such that $u(a)=1$ and $u(b)=0$. But then $\varphi_{u}\left(w_{u}\right)(a) \neq \varphi_{u}\left(w_{u}\right)(b)$, since $w_{u} \in N w_{u}$. Therefore $K\left(\varphi_{u}\right)(a) \neq K\left(\varphi_{u}\right)(b)$. Hence

$$
f:=\prod\left\{K\left(\varphi_{u}\right) \mid u \in Y\right\}: K(Y) \rightarrow \prod\left\{K\left(N w_{u}\right) \mid u \in Y\right\}
$$

is an embedding, whence $K(Y) \in \mathbb{S} \mathbb{P}(\mathbb{H}(K(W)))=\mathscr{B}$.

Conversely, we assume that $A=K(Y) \in \mathscr{B}$. We shall show that $Y$ is the union of the subspaces $Y^{w}(w \in W)$. By Lemma 3.8, $Y \in \mathscr{Y}^{\mathscr{B}}$ implies that

$$
Y=\bigcup\left\{\operatorname{im} \Phi_{w} \mid w \in W\right\}
$$

Now let $y=w \circ x \in \operatorname{im} \Phi_{w}$. We have, remembering that $x$ is a $\mathscr{Y}^{\mathscr{B}}$-morphism and 
that we have identified $K H(B)$ with $B$, that for any $a \in K(Y)$,

$$
\begin{aligned}
g_{\mu}(y)(a) & =\left(g_{\mu}(w \circ x)\right)(a) \\
& = \begin{cases}(w \circ x)\left(f_{\mu}^{+}(a)\right) & \text { if } \mu \in N^{+} \\
1-(w \circ x)\left(f_{\mu}^{-}(a)\right) & \text { if } \mu \in N^{-}\end{cases} \\
& = \begin{cases}w\left(x\left(f_{\mu}^{+}(a)\right)\right) & \text { if } \mu \in N^{+}, \\
1-\left(w\left(x\left(f_{\mu}^{-}(a)\right)\right)\right) & \text { if } \mu \in N^{-}\end{cases} \\
& = \begin{cases}w\left(f_{\mu}^{+}(x(a))\right) & \text { if } \mu \in N^{+}, \\
1-\left(w\left(f_{\mu}^{-}(x(a))\right)\right) & \text { if } \mu \in N^{-}\end{cases} \\
& = \begin{cases}f_{\mu}^{+}(x(a))(w) & \text { if } \mu \in N^{+}, \\
1-f_{\mu}^{-}(x(a))(w) & \text { if } \mu \in N^{-}\end{cases} \\
& =x(a)\left(g_{\mu}(w)\right) .
\end{aligned}
$$

We claim that $\sigma_{w}(y)$ holds. Suppose that $\nu_{1}, \nu_{2} \in N$ are such that $g_{\nu_{1}}(w) \leqslant g_{\nu_{2}}(w)$. From above,

$$
\begin{array}{lll}
g_{v_{1}}(y) \leqslant g_{\nu_{2}}(y) \quad \text { if and only if } & (\forall a \in A) g_{\nu_{1}}(y)(a) \leqslant g_{\nu_{2}}(y)(a) \\
& \text { if and only if } \quad(\forall a \in A) x(a)\left(g_{v_{1}}(w)\right) \leqslant x(a)\left(g_{\nu_{2}}(w)\right) \\
& \text { if } \quad(\forall b \in K(W)) b\left(g_{v_{1}}(w)\right) \leqslant b\left(g_{\nu_{2}}(w)\right) \\
& \text { if and only if } \quad & g_{\nu_{1}}(w) \leqslant g_{v_{2}}(w),
\end{array}
$$

as required.

It was appropriate to include the preceding result here, since we had the necessary machinery assembled. We shall not need to use it in this paper. However we note that it plays a key role in [29].

\section{Natural dualities}

In this section we present a natural duality for any class $\| \mathbb{S P}(\underline{P})$, where $\underline{P}=\underline{P}^{\mathrm{N}}$ $(\mathbf{N} \in \mathscr{N})$. Classes of this form include those varieties $\mathbb{H} \mathbb{S P}(\underline{P})$ for which $\mathbb{N} \mathbb{S P}(\underline{P})=$ $\| S \mathbb{P}(\underline{P})$, and even where this fails, allow us to describe the free algebras in $H S \mathbb{P}(\underline{P})$. In many cases the duality for $\mathbb{I S P}(P)$ can be based on the piggyback method in its original form, as presented in [21, 20] (or see [15]). Such varieties include $\mathbf{P}_{m, n}, \mathbf{S}$ and MS. We shall give necessary and sufficient conditions on $\mathbf{N}$ for simple piggybacking to suffice, and show that this happens if and only if coproducts in $\mathrm{M} \mathbb{S P}(\underline{P})(=\| \mathbb{S P}(\underline{P}))$ are D-coproducts with a suitable $\mathbf{N}$-action. 
In certain cases the generalised piggyback method from [17] is needed. Indeed, it was an analysis of the variety $\mathbf{K}$ which led to the theory in [17]. This theory, as it applies to distributive-lattice-ordered algebras, has been presented many times, in survey form in [26], and, for particular varieties of the type we are considering here, in $[17,1]$. We shall not repeat the definitions here. We apply the Generalised Piggyback Duality Theorem in its brute force 'multiple algebra, single carrier' formulation, using copies of $\underline{P}$ indexed by $N$. This is more convenient for translating to the Priestley duality than the equivalent 'single algebra, multiple carrier' version. In [31] we discuss arbitrary varieties $\mathbb{H S \mathbb { P }}(\underline{\Pi})$, for $\underline{\Pi}$ a set of subdirectly irreducible algebras in $\mathscr{A}^{\mathrm{N}}$. In this situation a 'multiple algebra, multiple carrier' approach is required.

To aid readers not already familiar with [17] we treat simple piggybacking first.

THEOREM 4.1 (Simple Piggyback Duality Theorem, for distributive-lattice-ordered algebras). Suppose that $\mathscr{A}=1 \mathbb{S P}(\underline{P})$, where $\underline{P}$ is a finite algebra having a $\mathrm{D}$-reduct. Let $\alpha$ be a fixed element of $H(P)$. Let $\underset{\sim}{P}=(P ; \tau, R)$ be the topological relational structure in which

(i) $\tau$ is the discrete topology,

(ii) $R=S \cup T$, where

(a) $S$ is the collection of maximal $\mathscr{A}$-subalgebras of sublattices of the form

$$
\alpha^{-1}(\leqslant):=\left\{(a, b) \in \underline{P}^{2} \mid \alpha(a) \leqslant \alpha(b)\right\}, \quad \text { and }
$$

(b) $T$ is the set of graphs of a subset $\mathscr{E}$ of $\operatorname{End}(\underline{P})$.

Let $\mathscr{X}:=\square \mathbb{S}_{c} \mathbb{H}(\underset{\sim}{P})$. Assume that the following separation condition $(\mathrm{S})$ is satisfied:

(S) given $a, b \in \underline{P}$ with $a \neq b$, there exists $u \in \mathscr{E}$ such that $\alpha(u(a)) \neq$ $\alpha(u(b))$.

Then the hom-functors $D:=\mathscr{A}(-, \underline{P})$ and $E:=\mathscr{X}(-, \underline{\sim})$ set up a duality between $\mathscr{A}$ and $\mathscr{X}$.

Our candidate for $\alpha$ will be our right identity $e$ in $\mathbf{N}$. We begin by applying the theorem in the simplest case of all, when $\mathbf{N} \in \mathscr{N}$ and $N$ is discretely ordered. This means that the D-reduct of $\underline{P}=K(\mathbf{N})$ is the Boolean lattice $\underline{\mathbf{2}}^{N}$. We write a typical element $a \in 2^{N}$ as $\left\langle a_{v}\right\rangle$, where $a_{v}=a(\nu)(v \in N)$. In examples, we take a fixed listing of the elements of $N$ and write $\left\langle a_{1}, a_{2}, \ldots, a_{k}\right\rangle$ more compactly as the binary string $a_{1} a_{2} \ldots a_{k}$. In view of the distinguished, schizophrenic, status we give to $2^{N}$ we shall adopt special symbols $\varphi_{\mu}^{ \pm}$and $\gamma_{\mu}$ for the operations instead of the generic $f_{\mu}^{ \pm}$ and $g_{\mu}$.

We make $\mathbf{2}^{N}$ into an object $\underline{\mathbf{2}}^{N}$ in $\mathbf{D}-\mathbf{N}$ as follows. Define operations $\varphi_{\mu}^{+}\left(\mu \in N^{+}\right)$ 
and $\varphi_{\mu}^{-}\left(\mu \in N^{-}\right)$by

$$
\begin{cases}\varphi_{\mu}^{+}\left(\left\langle a_{v}\right\rangle\right)=\left\langle a_{\mu \nu}\right\rangle & \left(\mu \in N^{+}\right) \\ \varphi_{\mu}^{-}\left(\left\langle a_{v}\right\rangle\right)=\left\langle\overline{a_{\mu \nu}}\right\rangle & \end{cases}
$$

where $\bar{\delta}=1-\delta$ for $\delta=0,1$. In the case of the Ockham variety $\mathbf{P}_{m, n}$, where $N=\left\{1, \gamma, \ldots, \gamma^{m-1}\right\}$ and $\gamma^{m}=\gamma^{n}$, we have the formula $\sim a=\varphi_{\gamma}\left(\left\langle a_{v}\right\rangle\right)=\left\langle\overline{a_{\gamma v}}\right\rangle$, as given in [17].

We check now that the algebraic structure of $\underline{P}$, in which the operations are dual to the operations $g_{\mu}$ given by the left action of $N$ on itself, is exactly that we have defined on $\underline{\mathbf{2}}^{N}$, when $\left\langle a_{v}\right\rangle$ stands for $\langle a(\nu)\rangle$. We have $\varphi_{\mu}^{ \pm}$given by

$$
\begin{cases}\varphi_{\mu}^{+}(a)(\nu)=a(\mu \nu)=a\left(g_{\mu}(\nu)\right) & \text { if } \nu \in N^{+}, \\ \varphi_{\mu}^{-}(a)(\nu)=\overline{a(\mu \nu)}=\overline{a\left(g_{\mu}(\nu)\right)} & \text { if } \nu \in N^{-},\end{cases}
$$

which agrees with the definition of $\varphi_{\mu}^{ \pm}$given by 2.6 .

The same set $2^{N}$ carries an alternative structure, in the following way. We define

$$
a \preccurlyeq b \text { if and only if } \begin{cases}a_{v}=1 \text { implies } b_{v}=1 & \text { if } v \in N^{+}, \\ b_{v}=1 \text { implies } a_{v}=1 & \text { if } v \in N^{-}\end{cases}
$$

This is a partial order on $2^{N}$, which we refer to as the \pm -order. In the case of the Ockham monoid $\mathbf{N}_{m, n}$ it is called the alternating order. We also define operations $\gamma_{\mu}(\mu \in N)$ as follows: $\gamma_{\mu}\left(\left\langle a_{\nu}\right\rangle\right):=\left\langle a_{\nu \mu}\right\rangle$. For $a=\left\langle a_{\nu}\right\rangle, b=\gamma_{\mu}(a)$ we have $b(v)=1$ if and only if $a(v \mu)=1$. Therefore for $\lambda, \mu, v \in N$ we have

$$
\begin{aligned}
\gamma_{\lambda}\left(\gamma_{\mu}(a)\right)(\nu)=1 & \text { if and only if } \gamma_{\mu}(a)(\nu \lambda)=1 \\
& \text { if and only if } a(\nu \lambda \mu)=1 \\
& \text { if and only if }\left(\gamma_{\lambda \mu}(a)\right)(\nu)=1 .
\end{aligned}
$$

Hence $\gamma_{\lambda} \circ \gamma_{\mu}=\gamma_{\lambda \mu}$. Also, for any $\mu \in N^{+}$,

$$
\begin{gathered}
\left\langle a_{\nu}\right\rangle \preccurlyeq\left\langle b_{\nu}\right\rangle \text { implies } \begin{cases}a_{v} \leqslant b_{v} & \text { if } v \in N^{+}, \\
a_{\nu} \geqslant b_{\nu} & \text { if } \nu \in N^{-}\end{cases} \\
\text {implies } \begin{cases}a_{v \mu} \leqslant b_{v \mu} & \text { if } v \in N^{+}, \\
a_{v \mu} \leqslant b_{v \mu} & \text { if } v \in N^{-}\end{cases} \\
\text {implies }\left\langle a_{\nu \mu}\right\rangle \preccurlyeq\left\langle b_{v \mu}\right\rangle .
\end{gathered}
$$

Hence $\gamma_{\mu}$ preserves $\preccurlyeq$ if $\mu \in N^{+}$, and, likewise, $\gamma_{\mu}$ reverses $\preccurlyeq$ if $\mu \in N^{-}$. Therefore $\left(2^{N} ; \tau, \preccurlyeq,\left\{\gamma_{\mu}\right\}_{\mu \in N}\right)$ becomes a space in N-P. 
Summing up, we have two structures with underlying set $2^{N}$ :

$$
\begin{aligned}
& \underline{\mathbf{2}}^{N}:=\left(2^{N} ; \wedge, \vee, 0,1,\left\{\varphi_{\mu}^{+}\right\}_{\mu \in N^{+}},\left\{\varphi_{\mu}^{-}\right\}_{\mu \in N^{-}}\right) \in \mathbf{D}-\mathbf{N}, \\
& \stackrel{2}{ }^{N}:=\left(2^{N} ; \tau, \preccurlyeq,\left\{\gamma_{\mu}\right\}_{\mu \in N}\right) \in \mathbf{N}-\mathbf{P},
\end{aligned}
$$

the first of which is the original algebra $\underline{P}$. There should not be any confusion with the usage here and that in Section 2, where $\mathbf{2}$ denoted the 2-element chain in $\mathbf{P}$.

The following duality theorem, based on these objects, generalises that obtained for $\mathbf{P}_{m, n}$ in [20], and applies to any of the Cornish varieties $\mathbf{D}-\mathbf{N}$ ( $\mathbf{N}$ a \pm -monoid).

THEOREM 4.2. Let $\mathbf{N} \in \mathscr{N}$ be a weak \pm -monoid. Let $\mathscr{A}:=\mathbb{H} \mathbb{S P}\left(\underline{\mathbf{2}}^{N}\right)$ and $\mathscr{X}:=\mathbb{\square} \mathbb{S}_{c} \mathbb{P}\left(2^{N}\right)$. Then the hom-functors $D$ and $E$ given on objects by

$$
\begin{aligned}
& D: A \mapsto \mathscr{A}\left(A, \underline{\mathbf{2}}^{N}\right) \leq \mathbf{2}^{N} \in \mathscr{X}, \\
& E: X \mapsto \mathscr{X}\left(X, \underline{\mathbf{2}}^{N}\right) \leq \underline{\mathbf{2}}^{N} \in \mathscr{A}
\end{aligned}
$$

set up a natural duality between $\mathscr{A}$ and $\mathscr{X}$.

PROOF. Lemma 3.5 of [ 17$]$ implies that there exists a unique maximal $\mathscr{A}$-subalgebra $e^{-1}(\leqslant)^{o}$ of $e^{-1}(\leqslant)$ and that this is given by

$(a, b) \in e^{-1}(\leqslant)^{o}$ if and only if $e(f(a)) \leqslant e(f(b))$ for all unary operations $f$

if and only if $(\forall v \in N) \begin{cases}a\left(g_{v}(e)\right) \leqslant b\left(g_{v}(e)\right) & \text { if } v \in N^{+}, \\ a\left(g_{\nu}(e)\right) \geqslant b\left(g_{v}(e)\right) & \text { if } v \in N^{-}\end{cases}$

if and only if $(\forall v \in N) \begin{cases}a(v e) \leqslant b(v e) & \text { if } v \in N^{+}, \\ a(v e) \geqslant b(v e) & \text { if } v \in N^{-}\end{cases}$

if and only if $(\forall v \in N) \begin{cases}a(v) \leqslant b(v) & \text { if } v \in N^{+}, \\ a(v) \geqslant b(v) & \text { if } v \in N^{-}\end{cases}$

if and only if $a \preccurlyeq b$.

For the separation condition, (S), we use the fact that each map $\eta_{\mu}: v \mapsto v \mu$ is the dual of an endomorphism $u_{\mu} \in$ End $\underline{P}$; see Lemma 3.1. Given $a \neq b$ in $\underline{P}$ there exists $\mu \in N$ such that $\mu(a) \neq \mu(b)$. Then

$$
\begin{aligned}
e\left(u_{\mu}(a)\right) & =\left(\eta_{\mu}(e)\right)(a)=(e \mu)(a) \\
& =\mu(a) \\
& \neq \mu(b) \\
& =(e \mu)(b)=\left(\eta_{\mu}(e)\right)(b)=e\left(u_{\mu}(b)\right) .
\end{aligned}
$$

Hence (S) holds. (Note that we have used here the fact that $e$ is a left identity in the monoid N.) 
There are other varieties $\mathscr{A}^{\mathrm{N}}$ which come within the scope of the Simple Piggyback Duality Theorem besides those covered by Theorem 4.2. We shall work towards identifying these, at the same time preparing for the application of the Generalised Piggyback Duality Theorem to arbitrary quasivarieties $0 S \mathbb{P}(\underline{P})$.

TheOREM 4.3 (Generalised Piggyback Duality Theorem, for distributive-latticeordered algebras). Suppose that $\mathscr{A}=\mathbb{\mathbb { S }} \mathbb{P}(\underline{\Pi})$, where $\underline{\Pi}$ is a finite set of finite algebras of a given fixed type each having a D-reduct. For each $\underline{P}$ in $\underline{\Pi}$ let $\Omega_{\underline{P}}$ be a (possibly empty) subset of $\mathbf{D}(\underline{P}, \underline{\mathbf{2}})$.

Let $\underset{\sim}{\sim}=(\Pi ; \tau, R)$ be the topological relational structure on $\bigcup\{P \mid \underline{P} \in \underline{\Pi}\}$ in which

(i) $\tau$ is the discrete topology,

(ii) $R=S \cup T$, where

(a) $S$ is the collection of maximal $\mathscr{A}$-subalgebras of sublattices of the form

$$
(\alpha, \beta)^{-1}(\leqslant):=\{(a, b) \in P \times Q \mid \alpha(a) \leqslant \beta(b)\},
$$

where $\alpha \in \Omega_{\underline{p}}, \beta \in \Omega_{\underline{Q}}(\underline{P}, \underline{Q} \in \underline{\Pi})$, and

(b) $T$ is the set of graphs of a set $\mathscr{E} \subseteq \bigcup\{\mathscr{A}(\underline{P}, \underline{Q}) \mid \underline{P}, \underline{Q} \in \underline{\Pi}\}$ of endomorphisms satisfying the following separation condition $(\mathrm{S})$ :

for all $\underline{P} \in \underline{\Pi}$, given $a, b \in \underline{P}$ with $a \neq b$, there exist $\underline{Q} \in \underline{\Pi}, u \in$ $\mathscr{A}(\underline{P}, \underline{Q}) \cap \mathscr{E}$ and $\alpha \in \Omega_{\underline{Q}}$ such that $\alpha(u(a)) \neq \alpha(u(b))$.

Then $R$ yields a duality on $\mathscr{A}$.

In the statement of the theorem we have used terminology adopted since [17] was written. When we say that the set $R$ yields a duality on $\mathscr{A}$ we mean that the structure $\underset{\sim}{ }$ generates a class $\mathscr{X}:=\mathbb{} \mathbb{S}_{c} \mathbb{P}(\underset{\sim}{\Pi})$ of multi-sorted structures such that the natural hom-functors $D$ and $E$ into $\underline{\Pi}$ and $\underset{\sim}{\sim}$ set up a dual equivalence, as described in [17] or in [26].

We want to apply this theorem in the case that $\mathscr{A}=\mathbb{U S \mathbb { P }}(\underline{P})$, where $\underline{P}$ is of the form $\underline{P}^{\mathrm{N}}$. The separation condition (S) in 4.3 is automatically satisfied if we take $\underline{\Pi}$ to consist of copies $\underline{P}_{\alpha}$ of $\underline{P}$ indexed by the points $\alpha$ of $N$ and, for each $\alpha \in N$, take $\Omega_{\underline{P}_{\alpha}}=\{\alpha\}$, where $\alpha$ is here regarded as an element of $N_{\alpha}:=H\left(P_{\alpha}\right)$. (Alternatively we can take $\underline{\Pi}$ to contain the single algebra $\underline{P}$, and take $\Omega_{\underline{P}}=N$.) The next lemma tells us when we can satisfy (S) more economically. The endomorphisms of $\underline{P}$ were described in Lemma 3.1. We shall define

$$
N_{\text {End }}:=\left\{\mu \in N \mid \eta_{\mu} \text { is order-preserving }\right\} .
$$

LEMma 4.4. Let $\mathbf{N} \in \mathscr{N}$. Let $C \subseteq N$ be such that $N=C N_{\text {End. Then for } a \neq b}$ there exists $\alpha \in C$ and $v \in N_{\text {End }}$ such that $\alpha\left(u_{v}(a)\right) \neq \alpha\left(u_{v}(b)\right)$. Conversely, if this 
separation condition is to be satisfied, then the set $C$ of points $\alpha$ required above is such that $N=C N_{\text {End }}$.

PROOF. For $a \in \underline{P}$ and $\nu \in N_{\text {End }}, \alpha\left(u_{v}(a)\right)=\left(\eta_{v}(\alpha)\right)(a)=(\alpha v)(a)$. Since every element of $N$ is expressible in the form $\alpha v$, where $\alpha \in C$ and $v \in N_{\text {End }}$, the first part follows.

Conversely, for any $\mu \in N$, let $a, b \in P$ be defined by $a(\nu)=1$ if and only if $v \geqslant \mu$ and $b(v)=1$ if and only if $v>\mu$. Then $a$ and $b$ are distinct, and the only element $\lambda \in N$ for which $\lambda(a) \neq \lambda(b)$ is $\lambda=\mu$. To satisfy the separation condition we therefore have to be able to express $\mu$ as a product $\alpha \nu$, where $\alpha \in C, \nu \in N_{\text {End }}$.

As was revealed in [17], there is an interaction between the separation condition (S), joint-ontoness of the maps $\Phi_{\alpha}$ (as in 3.9, and the choice of carrier sets $\Omega_{\underline{P}}$ for $\underline{P} \in \underline{\Pi}$. To explore this, we first give a lemma characterising im $\Phi_{\alpha}$, generalising [17, Lemma 3.3].

LEMMA 4.5. Let $\mathbf{N} \in \mathscr{N}$ and let $\mathscr{A}=\| \mathbb{S P}(\underline{P})$. For each $\alpha \in N$ take $\underline{P}_{\alpha}$ to be $a$ copy of $\underline{P}$, and regard $\alpha$ as an element of $H\left(P_{\alpha}\right)$. Define maps

$$
\begin{aligned}
& \Phi_{\alpha}:=\alpha \circ-: \mathscr{A}\left(A, \underline{P}_{\alpha}\right) \rightarrow H(A)_{\alpha}, \\
& \Theta_{\alpha}:=k_{A}(-) \circ h_{\alpha}: H(A)_{\alpha} \rightarrow \mathscr{A}\left(A, \underline{P}_{\alpha}\right),
\end{aligned}
$$

where

$$
H(A)_{\alpha}:=\left\{y \in H(A) \mid \exists \varphi \in \mathscr{Y}^{\mathscr{\alpha}}\left(N_{\alpha}, H(A)\right) \varphi(\alpha)=y\right\}
$$

and $h_{\alpha}(y)(\nu):=g_{\nu}(y)$. Then $\Phi_{\alpha}$ and $\Theta_{\alpha}$ are mutually inverse bijections, for each $\alpha$. Furthermore, for each $\alpha$ and each $A, H(A)_{\alpha} \subseteq H(A)^{\alpha}$.

PROOF. We do not suppress the isomorphism $k_{A}: A \rightarrow K H(A)$ in this proof and we write $N_{\alpha}=\left\{v^{\alpha} \mid v \in N\right\}$. Let $s_{\alpha}$ be the natural bijection from $\mathscr{Y}\left(N_{\alpha}, H(A)\right)$ to $\mathscr{A}\left(A, \underline{P}_{\alpha}\right)$ and $t_{\alpha}$ that from $\mathscr{Y}\left(N_{\alpha}, H(A)\right)$ to $H(A)_{\alpha}$ :

$$
\left(\forall \varphi \in \mathscr{Y}\left(N_{\alpha}, H(A)\right)\right) \quad s_{\alpha}(\varphi)=k_{A}(-) \circ \varphi
$$

and $t_{\alpha}(\varphi):=\varphi(\alpha)$.

We now need $\Phi_{\alpha} \circ s_{\alpha}=t_{\alpha}$ and $\Theta_{\alpha} \circ t_{\alpha}=s_{\alpha}$. For any $a \in A$ and $\varphi \in$ $\mathscr{Y}\left(N_{\alpha}, H(A)\right)$,

$$
\left(\Phi_{\alpha}\left(s_{\alpha}(\varphi)\right)\right)(a)=\left(s_{\alpha}(\varphi)(a)\right)(\alpha)=\varphi(\alpha)(a)=t_{\alpha}(\varphi)(a) .
$$

This shows that $\Phi_{\alpha} \circ s_{\alpha}=t_{\alpha}$. For any $\nu \in N$ and any $\varphi \in \mathscr{Y}\left(N_{\alpha}, H(A)\right)$,

$$
\left(h_{\alpha} \circ t_{\mu}\right)(\varphi)\left(v^{\alpha}\right)=\left(h_{\alpha}(\varphi(\alpha))\right)\left(v^{\alpha}\right)=g_{v}(\varphi(\alpha))=\varphi\left(v^{\alpha}\right)
$$




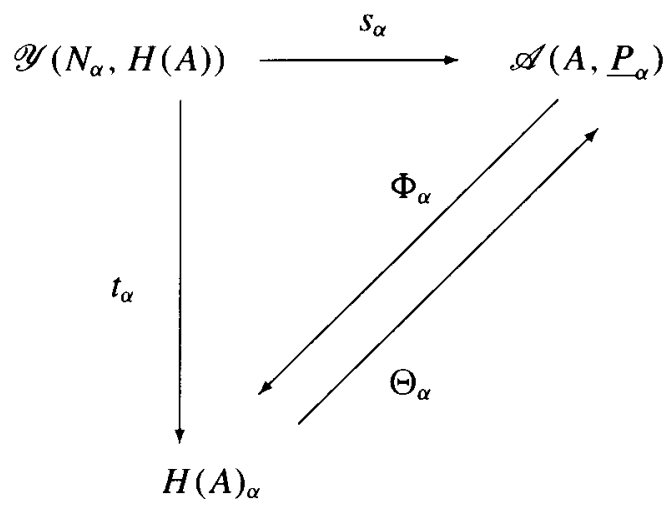

Hence $h_{\alpha} \circ t_{\alpha}$ is the identity on $\mathscr{Y}\left(N_{\alpha}, H(A)\right)$, whence $\Theta_{\alpha} \circ t_{\alpha}=s_{\alpha}$.

Furthermore, for each $\alpha$,

$$
H(A)_{\alpha}=\operatorname{im} \Phi_{\alpha}=\left\{\varphi(\alpha) \mid \varphi \in \mathscr{Y}^{\mathscr{A}}\left(H\left(P_{\alpha}\right), H(A)\right)\right\} .
$$

We now claim that $\operatorname{im} \Phi_{\alpha} \subseteq H(A)^{\alpha}$ : let $\nu_{1}, v_{2} \in N$ and suppose $\nu_{1} \alpha \leqslant v_{2} \alpha$ in $N$. Then $v_{1} \varphi(\alpha)=\varphi\left(v_{1} \alpha\right) \leqslant \varphi\left(v_{2} \alpha\right)=v_{2} \varphi(\alpha)$.

We can now derive the following proposition.

Proposition 4.6. Let $\mathbf{N} \in \mathscr{N}$ be a monoid with identity e and let $\mathscr{A}:=\mathbb{1 S} \mathbb{P}(\underline{P})$, where $\underline{P}:=\underline{P}^{\mathrm{N}}$. Then the following conditions are equivalent:

(i) there exists $\alpha \in N$ such that $N=\alpha N_{\text {End; }}$;

(ii) there exists $\alpha \in N$ such that for $a \neq b$ in $\underline{P}$, there exists $u \in$ End $\underline{P}$ such that $\alpha(u(a)) \neq \alpha(u(b))$;

(iii) $N=N_{\text {End }}$, that is, for each $\mu \in N, \nu_{1} \leqslant \nu_{2}$ implies $\nu_{1} \mu \leqslant \nu_{2} \mu$;

(iv) for $a \neq b$ in $\underline{P}$, there exists $u \in$ End $\underline{P}$ such that $e(u(a)) \neq e(u(b))$;

(v) $\Phi_{e}: \mathscr{A}(A, \underline{P}) \rightarrow H(A)$ is surjective for every $A \in \mathscr{A}$;

(vi) for all $A \in \mathscr{A}$,

$$
H(A) \models(\forall y)\left(\left(\nu_{1} \leqslant \nu_{2} \text { implies } g_{v_{1}}(y) \leqslant g_{v_{2}}(y)\right) .\right.
$$

Furthermore, if (i)-(vi) hold then $\mathbb{\triangle S P}(\underline{P})=\mathbb{H} \mathbb{S P}(\underline{P})$.

Proof. By Lemma 4.4, (i) is equivalent to (ii) and (iii) is equivalent to (iv), the latter using the fact that $e$ is a left identity. Assume that (i) holds. Then

$$
|N|=\left|\alpha N_{\text {End }}\right| \leqslant\left|N_{\text {End }}\right| \leqslant|N| .
$$


We deduce that (i) implies (iii), and (iii) implies (i) trivially. Therefore (i)-(iv) are equivalent. The equivalence of (iv) and (v) comes from [17, Lemma 2.4].

If $\Phi_{e}$ is surjective for every $A$, then, by Lemma 4.5 ,

$$
H(A)=H(A)^{e}=\left\{y \in H(A) \mid \nu_{1} \leqslant v_{2} \text { implies } g_{v_{1}}(y) \leqslant g_{v_{2}}(y)\right\} .
$$

Hence (v) implies (vi). To prove that (vi) implies (iii) take $A=\underline{P}$, so that $H(A)=\mathbf{N}$.

For the final statement, note that if the variety and quasivariety do not coincide then we can find a subdirectly irreducible algebra $Q$ which is a homomorphic image of $\underline{P}$ but not isomorphic to a subalgebra of $\underline{P}$. This means that there is a point $\kappa \in N$ such that there is no $\mathbf{N}$-P-morphism from $N$ onto $N \kappa$. In particular $\eta_{\kappa}$ is not such a morphism, so (i) cannot hold.

The conditions in the preceding proposition are exactly those required for simple piggybacking to work for $\mathbb{U S P}(\underline{P})$. The schizophrenic object for this has $P$ as its underlying set. This set sits inside $2^{N}$ as the order-preserving maps from $\underline{P}$ to $\underline{\mathbf{2}}$. In fact the objects $\underline{P}$ and $\underset{\sim}{P}$ inherit their structure from $\underline{\mathbf{2}}^{N}$ and ${\underset{\sim}{2}}^{N}$, as the following lemma implies. For the Ockham case, see [17, Proposition 3.14].

LEMMA 4.7. Let $\mathbf{N} \in \mathscr{N}$ be a monoid with identity e. Then

$$
\underline{P}=\left\{a: N \rightarrow 2 \mid \underline{\mathbf{2}}^{N} \models \sigma_{e}(a)\right\} \leq \underline{\mathbf{2}}^{N},
$$

where

$$
\sigma_{e}(a):=\bigwedge\left\{v_{1} \leqslant v_{2} \Rightarrow \gamma_{\nu_{1}}(a) \preccurlyeq \gamma_{\nu_{2}}(a) \mid v_{1}, \nu_{2} \in N\right\}
$$

Furthermore,

(i) for any $\mu \in N$, the map $\nu \mapsto v \mu$ is order-preserving if and only if $\gamma_{\mu}(P) \subseteq P$;

(ii) the unique maximal subalgebra $e^{-1}(\leqslant)^{o}$ of $e^{-1}(\leqslant)$ is $\preccurlyeq \cap P^{2}$.

PROOF. Suppose $a: N \rightarrow 2$ is order-preserving and suppose $v_{1} \leqslant v_{2}$. For $v \in N$,

$$
\begin{aligned}
& v_{1} \leqslant v_{2} \text { implies } \begin{cases}v v_{1} \leqslant v v_{2} & \text { if } v \in N^{+}, \\
v v_{1} \geqslant v v_{2} & \text { if } v \in N^{-}\end{cases} \\
& \text {implies } \begin{cases}a_{v v_{1}} \leqslant a_{v v_{2}} & \text { if } v \in N^{+}, \\
a_{v v_{1}} \geqslant a_{v v_{2}} & \text { if } v \in N^{-}\end{cases} \\
& \text {implies } \begin{cases}\gamma_{v_{1}}(a)(v) \leqslant \gamma_{v_{2}}(a)(v) & \text { if } v \in N^{+}, \\
\gamma_{v_{1}}(a)(v) \geqslant \gamma_{v_{2}}(a)(v) & \text { if } v \in N^{-}\end{cases} \\
& \text {implies } \gamma_{v_{1}}(a) \preccurlyeq \gamma_{v_{2}}(a) .
\end{aligned}
$$


Hence $a$ is such that ${\underset{2}{2}}^{N} \models \sigma_{e}(a)$.

Conversely, assume ${\underset{2}{ }}^{N} \models \sigma_{e}(a)$. Recall that $e \in N^{+}$. We have

$$
\begin{aligned}
\nu_{1} \leqslant v_{2} & \text { implies } \gamma_{\nu_{1}}(a) \preccurlyeq \gamma_{\nu_{2}}(a) \\
& \text { implies }\left\langle a_{\nu v_{1}}\right\rangle \preccurlyeq\left\langle a_{\nu v_{2}}\right\rangle \\
& \text { implies } a_{\nu_{1}}=a_{e \nu_{1}} \leqslant a_{e \nu_{2}}=a_{\nu_{2}} \\
& \text { if and only if } a\left(\nu_{1}\right) \leqslant a\left(v_{2}\right) .
\end{aligned}
$$

Now consider (i). Assume that $v \mapsto v \mu$ is order-preserving and let $a \in \underline{P}$. Then

$$
\begin{aligned}
v_{1} \leqslant v_{2} & \text { implies } v_{1} \mu \leqslant v_{2} \mu \\
& \text { implies }\left\langle a_{v v_{1} \mu}\right\rangle \preccurlyeq\left\langle a_{\nu \nu_{2} \mu}\right\rangle \\
& \text { implies } \gamma_{v_{1}}\left(\gamma_{\mu}(a)\right) \preccurlyeq \gamma_{v_{2}}\left(\gamma_{\mu}(a)\right) .
\end{aligned}
$$

Hence $\sigma_{e}\left(\gamma_{\mu}(a)\right)$ holds, so $\gamma_{\mu}(a) \in P$. Conversely, suppose we have $\gamma_{\mu}(P) \subseteq P$. This means that for every order-preserving map $a: N \rightarrow \underline{\mathbf{2}}, \gamma_{\mu}(a)$ is also orderpreserving. Take $\nu_{1} \leqslant \nu_{2}$ in $N$. We have $\nu_{1} \mu \leqslant \nu_{2} \mu$ if and only if $a\left(\nu_{1} \mu\right) \leqslant a\left(\nu_{2} \mu\right)$ for all $a \in P$, that is, if and only if $\gamma_{\mu}(a)\left(v_{1}\right) \leqslant \gamma_{\mu}(a)\left(\nu_{2}\right)$.

Finally, (ii) is proved exactly as in 4.2 .

As a corollary we have the following generalisation of Theorem 4.2.

THEOREM 4.8. Assume that the equivalent conditions of Proposition 4.6 hold. Then $\square S \mathbb{P}(\underline{P})$ has a natural duality based on the single algebra $\underline{P}$ and single carrier $\alpha$, where $\alpha$ is the identity $e$ of the monoid $N$. In this duality, the relations are the \pm -order $\preccurlyeq$ on $P$ (the unique maximal subalgebra of $e^{-1}(\leqslant)$ ) and the graphs of the endomorphisms $\left\{u_{\mu}\right\}_{\mu \in N}$.

4.9. Example. Double MS-algebras. Take $\mathbf{N}$ to be the monoid $\mathbf{N}^{\mathrm{DMS}}$ as in $2.4(2)$, 3.7(1). From the facts assembled there we see, from Proposition 4.6, that Theorem 4.8 applies.

We have $P$ as the set of quintuples $\left\langle a_{h^{2}}, a_{h}, a_{1}, a_{g}, a_{g^{2}}\right\rangle$ in which $a_{h^{2}} \leqslant a_{1} \leqslant a_{g^{2}}$ and $a_{h} \leqslant a_{g}$. We shall represent these 12 elements as binary strings of length 5 . The algebra $\underline{P}$ is as shown in Figure 10(a). The same set under its \pm -order is shown in Figure 10(b). Here we have a \pm -monoid, and hence only a single operation assigned to each member of $N$. The operations are given by

$$
\begin{aligned}
& \varphi_{g}\left(\left\langle a_{h^{2}}, a_{h}, a_{1}, a_{g}, a_{g^{2}}\right\rangle\right)=\left\langle\overline{a_{g}}, \overline{a_{g^{2}}}, \overline{a_{g}}, \overline{a_{g^{2}}}, \overline{a_{g}}\right\rangle, \\
& \varphi_{h}\left(\left\langle a_{h^{2}}, a_{h}, a_{1}, a_{g}, a_{g^{2}}\right\rangle\right)=\left\langle\overline{a_{h}}, \overline{a_{h^{2}}}, \overline{a_{h}}, \overline{a_{h^{2}}}, \overline{a_{h}}\right\rangle .
\end{aligned}
$$


LEMMA 4.11. Under the assumptions of Theorem 4.10,

$$
\left(\left\langle a_{\nu}^{\alpha}\right\rangle,\left\langle b_{v}^{\beta}\right\rangle\right) \in(\alpha, \beta)^{-1}(\leqslant)^{o} \text { if and only if } q_{\alpha}\left(\left\langle a_{v}^{\alpha}\right\rangle\right) \preccurlyeq q_{\beta}\left(\left\langle b_{\nu}^{\beta}\right\rangle\right)
$$

and $q_{\beta} \circ \iota_{\alpha, \beta} \circ g_{\alpha}=\gamma_{\beta} \circ q_{\alpha}$.

ProOF. Let $a=\left\langle a_{v}^{\alpha}\right\rangle \in \underline{P}_{\alpha}, b=\left\langle b_{v}^{\beta}\right\rangle \in \underline{P}_{\beta}$. Then, arguing as in the proof of 4.2 .

$$
\begin{aligned}
& (a, b) \in(\alpha, \beta)^{-1}(\leqslant)^{o} \\
& \text { if and only if }(\forall v \in N) \begin{cases}\left(g_{v}(\alpha)\right)(a) \leqslant\left(g_{\nu}(\beta)\right)(b) & \text { if } \nu \in N^{+}, \\
\left(g_{v}(\alpha)\right)(a) \geqslant\left(g_{\nu}(\beta)\right)(b) & \text { if } \nu \in N^{-}\end{cases} \\
& \text {if and only if }(\forall v \in N) \begin{cases}a(v \alpha) \leqslant b(v \beta) . & \text { if } v \in N^{+}, \\
a(v \alpha) \geqslant b(\nu \beta) & \text { if } v \in N^{-} .\end{cases}
\end{aligned}
$$

Also

$$
\begin{aligned}
q_{\beta}\left(\iota_{\alpha, \beta}\left(g_{\alpha}\left(\left\langle a_{\nu}^{\alpha}\right\rangle\right)\right)\right) & =q_{\beta}\left(\iota_{\alpha, \beta}\left(\left\langle a_{\nu \alpha}^{\alpha}\right\rangle\right)\right) \\
& =q_{\beta}\left(\left\langle a_{\nu \alpha}^{\beta}\right\rangle\right) \\
& =\left\langle a_{\nu \alpha \beta}\right\rangle \\
& =\gamma_{\beta}\left(\left\langle a_{\nu \alpha}\right\rangle\right)=\gamma_{\beta}\left(q_{\alpha}\left(\left\langle a_{\nu}^{\alpha}\right\rangle\right)\right) .
\end{aligned}
$$

We conclude that $q_{\beta} \circ \iota_{\alpha, \beta} \circ g_{\alpha}=\gamma_{\beta} \circ q_{\alpha}$.

We may use the maps $q_{\alpha}$ simultaneously to embed all our copies $P_{\alpha}$ of $P$ into $2^{N}$. Lemma 4.11 says that $\bigcup \operatorname{im} q_{\alpha}$ becomes a subspace of ${\underset{\sim}{2}}^{N}$ in $\mathbf{N}$-P. The process we have carried out here on $\underset{\sim}{\Pi}$ is a special case of the translation process from a natural dual of an algebra to its Priestley dual, which we now outline. A more comprehensive account of this appears in [31], and, for the Ockham case which we generalise, in [17, Theorem 3.8].

Recall that the natural dual of an algebra $A \in \mathscr{A}$ is defined to be the disjoint union of the hom-sets $\mathscr{A}\left(A, \underline{P}_{\alpha}\right)$, structured pointwise by the relations described in Theorem 4.10. When $A=F \mathscr{A}(1)$, there is a bijective correspondence between $P_{\alpha}$ and $\mathscr{A}\left(A, \underline{P}_{\alpha}\right)$. Thus $\underset{\sim}{\prod}$, the disjoint union of the sets $P_{\alpha}$ equipped with the relational structure defined above, belongs to the dual category $\mathscr{X}$, and serves as the dual $D(F \mathscr{A}(1))$ of $F \mathscr{A}(1)$. See [17, Lemma 1.2].

Fix $A \in \mathscr{A}$. Define an equivalence relation $\rho$ on $D(A):=\bigcup_{\alpha \in N} \mathscr{A}\left(A, \underline{P}_{\alpha}\right)$ by $x \rho y$ if and only if $(x, y) \in \bigcup\left\{\operatorname{ker}(\alpha, \beta)^{o} \mid \alpha, \beta \in N\right\}$. 
Note that for $x \in \mathscr{A}\left(A, \underline{P}_{\alpha}\right), y \in \mathscr{A}\left(A, \underline{P}_{\beta}\right)$,

$$
\begin{aligned}
& x \rho y \text { if and only if }(x, y) \in \operatorname{ker}(\alpha, \beta)^{o} \\
& \text { if and only if }(\forall a \in A)(x(a), y(a)) \in \operatorname{ker}(\alpha, \beta)^{o} \\
& \text { if and only if }(\forall a \in A) \alpha(x(a))=\beta(y(a)) \\
& \text { if and only if } \alpha \circ x=\beta \circ y \\
& \text { if and only if } \Phi_{\alpha}(x)=\Phi_{\beta}(x),
\end{aligned}
$$

from which we see that $\rho$ is indeed an equivalence relation. The maps $\Phi_{\alpha}(\alpha \in N)$ map jointly onto $H(A)$, so that the map which sends $u \in H(A)$ to the equivalence class $x / \rho$, where $x \in D(A)$ is such that $u=\Phi_{\alpha}(x)$ for some $\alpha \in N$, is a well-defined bijection from $H(A)$ to $D(A) / \rho$. Also

$$
\begin{array}{rlll}
\Phi_{\alpha}(x) \leqslant \Phi_{\beta}(y) & \text { if and only if } & (\forall a \in A) \Phi_{\alpha}(x)(a) \leqslant \Phi_{\beta}(y)(a) \\
& \text { if and only if } & (\forall a \in A) \alpha(x(a)) \leqslant \beta(y(a)) \\
\text { if and only if } & (x, y) \in(\alpha, \beta)^{-1}(\leqslant)^{o} .
\end{array}
$$

Now consider the operations $g_{\mu}$ on $H(A)$. We have, using the fact that $x$ is a $\mathscr{Y}^{\mathscr{A}}-$ morphism,

$$
\begin{aligned}
& \Phi_{\beta}(y)=g_{\mu}\left(\Phi_{\alpha}(x)\right) \\
& \text { if and only if }(\forall a \in A) \Phi_{\beta}(y)(a)=\left(g_{\mu}\left(\Phi_{\alpha}\right)(x)\right)(a) \\
& \text { if and only if }(\forall a \in A) \begin{cases}\beta(y(a))=\alpha\left(x\left(\varphi_{\mu}^{+}(a)\right)\right) & \text { if } \mu \in N^{+}, \\
\beta(y(a))=1-\alpha\left(x\left(\varphi_{\mu}^{-}(a)\right)\right) & \text { if } \mu \in N^{-}\end{cases} \\
& \text {if and only if }(\forall a \in A) \begin{cases}\beta(y(a))=\alpha\left(\varphi_{\mu}^{+}(x(a))\right) & \text { if } \mu \in N^{+}, \\
\beta(y(a))=1-\alpha\left(\varphi_{\mu}^{-}(x(a))\right) & \text { if } \mu \in N^{-}\end{cases} \\
& \text {if and only if } \quad(\forall a \in A) \beta(y(a))=g_{v}(\alpha)(x(a)) \\
& \text { if and only if }(\forall a \in A) \beta(y(a))=(\mu \alpha)(x(a)) \\
& \text { if and only if } \Phi_{\beta}(y)=\Phi_{\mu \alpha}(x) \text {. }
\end{aligned}
$$

Hence $g_{\mu}\left(\Phi_{\alpha}(x)\right)=\Phi_{\mu \alpha}(x)$ for any $\mu, \alpha$ and $x$.

The topology on $D(A)$ is the sum topology on a disjoint union, from which $D(A) / \rho$ acquires its quotient topology. As in [17], the bijection from $D(A) / \rho$ to $H(A)$ is a homeomorphism.

As a special case, consider the object $\underset{\sim}{\Pi}$, which belongs to $\mathscr{X}$ and acts as $D(F \mathscr{A}(1))$. We regard this as a collection of copies of $P$. The statement and proof of the result below follow those of the special case given in [17, Theorem 3.15]. 
THEOREM 4.12. Let $\mathbf{N} \in \mathscr{N}$ and let $\mathscr{A}:=\square \mathbb{S P}\left(\underline{P}^{\mathbf{N}}\right)$. Then, for $s \geqslant 1$,

$$
\begin{aligned}
H(F \mathscr{A}(s)) & \cong\left\{\gamma_{\mu}(y)(\mu \in N) \mid\left(\mathbf{\sim}^{N}\right)^{s} \vDash \sigma_{e}(y)\right\}, \\
& \cong\left\{y \in\left(\mathbf{2}^{N}\right)^{s} \mid\left({\underset{\sim}{2}}^{N}\right)^{s} \models \sigma^{\mathrm{N}}(y)\right\} .
\end{aligned}
$$

PROOF. Since products in $\mathscr{X}$ are concrete products, it will suffice to consider $s=1$.

Carry out the translation process on $\prod_{\sim} \doteq D(F \mathscr{A}(1))$. We have that the algebra $\underline{P}$, regarded as a subset of $2^{N}$, consists exactly of those $y$ satisfying $\sigma_{e}(y)$, by Lemma 4.7 . The translation gives us an equivalence relation $\rho$ on $\underset{\sim}{\Pi}$ such that $\underset{\sim}{\Pi} / \rho$ is isomorphic in $\mathscr{Y}^{\mathscr{A}}$ to $H(F \mathscr{A}(1))$. Furthermore, by Lemma $4.11, \Pi / \rho$ can be identified, by means of the union of the maps $q_{\alpha}$, with the subset $\bigcup\left\{\gamma_{\alpha}(\widetilde{P}) \mid \alpha \in N\right\}$, with inherited $\preccurlyeq$-order and maps $\gamma_{\mu}(\mu \in N)$.

Now we wish to characterise this set as those $\gamma_{\alpha}(y)$ such that $2^{N} \models \sigma_{e}(y)(\alpha \in N)$. Note that the image of $P_{\mu}$ under $q_{\mu}$ is $\gamma_{\mu}(P)$. Since we know $P$ is characterised as those points $y$ in ${\underset{\sim}{2}}^{N}$ which satisfy $\sigma_{e}(y)$. This gives the first description of $H(F \mathscr{A}(1))$.

For the alternative description we have

$$
\begin{aligned}
\gamma_{\alpha}(P) & =\left\{\left\langle a_{v \alpha}\right\rangle \mid y=\left\langle a_{v}\right\rangle \text { satisfies } \sigma_{e}(y)\right\} \\
& =\left\{\left\langle a_{v \alpha}\right\rangle \mid v_{1} \leqslant v_{2} \text { implies } \gamma_{\nu_{1}}(y) \preccurlyeq \gamma_{v_{2}}(y)\right\} \\
& =\left\{\left\langle a_{v \alpha}\right\rangle \mid v_{1} \leqslant v_{2} \text { implies }\left\langle a_{v v_{1}}\right\rangle \preccurlyeq\left\langle a_{v v_{2}}\right\rangle\right\} \\
& =\left\{\left\langle a_{v \alpha}\right\rangle \mid v_{1} \alpha \leqslant v_{2} \alpha \text { implies }\left\langle a_{v v_{1} \alpha}\right\rangle \preccurlyeq\left\langle a_{v \nu_{2} \alpha}\right\rangle\right\} \\
& =\left\{\gamma_{\alpha}\left(\left\langle a_{v}\right\rangle\right) \mid v_{1} \alpha \leqslant v_{2} \alpha \text { implies } \gamma_{\nu_{1}}\left(\gamma_{\alpha}\left(\left\langle a_{v}\right\rangle\right) \preccurlyeq \gamma_{v_{2}}\left(\gamma_{\alpha}\left(\left\langle a_{v}\right\rangle\right)\right)\right\}\right. \\
& =\left\{\gamma_{\alpha}\left(\left\langle a_{v}\right\rangle\right) \mid \sigma_{\alpha}\left(\gamma_{\alpha}\left(\left\langle a_{v}\right\rangle\right)\right)\right\} .
\end{aligned}
$$

This completes the proof.

To find free algebras in practice we need not concern ourselves with simplifying our brute force duality by indexing the algebras $\underline{P}_{\alpha}$ by a minimal set of points $\alpha$ sufficient to achieve the separation condition. All we do is to calculate the sets $\gamma_{\alpha}(P)$, for all $\alpha \in N$, and to discard those which are not maximal with respect to inclusion.

Coproducts in general can be obtained in the same manner. Given a family $\left\{A_{i}\right\}_{i \in I}$ of algebras in $\amalg \mathbb{S P}(\underline{P})$, we form $H\left(\bigsqcup A_{i}\right)$ by taking the product in the dual category $\mathscr{X}$ of the family $\left\{D\left(A_{i}\right)\right\}$ (see [17]) and then applying the translation process.

It has long been known that coproducts in the variety $\mathbf{M}$ of de Morgan algebras are D-coproducts enriched with a (necessarily unique) de Morgan negation ([2, p. 214]). One way to prove this is to show that the natural and Priestley dualities for $\mathbf{M}$ are essentially the same (see [20]). We now present conditions for the two dualities for $\square \mathbb{S P}\left(\underline{P}^{\mathrm{N}}\right)$ to coincide.

THEOREM 4.13. Let $\mathbf{N} \in \mathscr{N}$ be a monoid with identity e, let $\underline{P}:=\underline{P}^{\mathrm{N}}$ and let $\mathscr{A}:=\mathbb{\triangle S P}(P)$. Then the following are equivalent: 
(i) every map $\eta_{\mu}: \nu \mapsto \nu \mu$ is order-preserving;

(ii) for every $A \in \mathscr{A}$, the map $\Phi_{e}: \mathscr{A}(A, \underline{P}) \rightarrow H(A)$ is a bijection;

(iii) $H(F \mathscr{A}(1))^{s}$ is isomorphic in $\mathbf{P}$ to $H(F \mathscr{A}(s))$ for $1 \leqslant s<\omega$;

(iv) products in $\mathscr{Y}^{\mathscr{A}}$ are concrete products;

(v) for any family $\left\{A_{i}\right\}_{i \in I}$ of algebras in $\mathscr{A}$, the D-reduct of $\mathscr{A}-\bigsqcup_{i \in I} A_{i}$ is the D-coproduct of the D-reducts of the algebras $A_{i}(i \in I)$.

If $($ i) $-(\mathrm{v})$ hold then $\operatorname{ISP}(\underline{P})=\mathbb{H} \mathbb{S} \mathbb{P}(\underline{P})$.

Proof. Conditions (i) and (ii) are equivalent by Proposition 4.6, and imply that $\mathscr{A}$ has a natural duality as in Theorem 4.8 . The translation process applied to this duality tells us that $\Phi_{e}$ sets up a bijection between the natural dual, $D(A)$, of $A \in \mathscr{A}$ and its Priestley dual $H(A)$. Since products in $\mathbf{P}$ are concrete, we conclude that (ii) implies (iv). Trivially (iv) implies (iii) and by Priestley duality (iv) is equivalent to (v).

Now assume that (i) fails, and assume that $\nu \mapsto \nu \lambda$ is not order-preserving. By the contrapositive of Lemma 4.7(i), $\gamma_{\lambda}(P) \nsubseteq P$. Take the brute force duality for $\mathscr{A}$ supplied by Theorem 4.10 , and apply the translation process to $\underset{\sim}{\Pi}$, embedding its components as the subsets $\gamma_{\mu}(P)$ of $2^{N}(\mu \in N)$ via the maps $q_{\mu}$ defined above. We have

$$
\begin{aligned}
H(F \mathscr{A}(s)) & \cong \bigcup_{\mu \in N}\left(\gamma_{\mu}(P)\right)^{s}, \\
H(F \mathscr{A}(1))^{s} & \cong \bigcup_{\mu_{1}, \ldots, \mu_{s} \in N} \gamma_{\mu_{1}}(P) \times \cdots \times \gamma_{\mu_{s}}(P) .
\end{aligned}
$$

Put $m=|P|$ and consider the $(m+1)$-tuple $\left(a_{1}, \ldots, a_{m}, b\right)$, where $a_{1}, \ldots, a_{m}$ are the distinct points of $P$ and $b \in \gamma_{\lambda}(P) \backslash P$. Because $\left|\gamma_{\mu}(P)\right| \leqslant|P|$ for all $\mu \in N$, we deduce that $\left(a_{1}, \ldots, a_{m}, b\right) \in H(F \mathscr{A}(1))^{m+1} \backslash H(F \mathscr{A}(m+1))$. Since also $H(F \mathscr{A}(m+1)) \subseteq H(F \mathscr{A}(1))^{m+1}$, these two dual spaces cannot be isomorphic posets. Thus (iii) fails. This completes the proof of the equivalence of (i)-(v).

Finally note that it was proved in 4.6 that (i) implies that $\mathbb{U S P}(\underline{P})=\mathbb{A S P}(P)$.

The varieties MS and DMS come within the scope of this theorem, and, more generally, the varieties $\mathbf{D M S}_{n}$ and $\mathbf{K}_{m, n}$ studied by M. Sequiera in [35] do too. We conclude with descriptions of free algebras for some other examples.

4.14. Example. Varieties generated by Boolean lattices. We generalise the wellknown characterisation of free de Morgan algebras. Suppose that $\mathbf{N}$ is any weak \pm -monoid, so that the dual algebra $\underline{P}:=\underline{P}^{N}$ is a Boolean lattice. Since the order is discrete, condition (i) in Theorem 4.13 holds. Take $\mathscr{A}:=\mathbb{H} \mathbb{S P}(\underline{P})$. Then $H(F \mathscr{A}(s))=\left(\mathbf{2}^{N}\right)^{s}$. Let $m=|N|, p=\left|N^{+} \cap N^{-}\right|$and $q=m-p$. The \pm -order on ${\underset{\sim}{2}}^{N}$ gives a poset $U$ which can be regarded as the product of $\overline{\mathbf{2}}^{p}$ and ${\underset{\sim}{q}}^{q}$ (where $\overline{\mathbf{2}}$ 
and $\underset{\sim}{2}$ denote respectively the 2-element antichain and the 2-element chain in $\mathbf{P}$ ). We see that $U$ is the disjoint union of $2 p$ copies of ${\underset{\sim}{2}}^{q}$ if $p>0$ and ${\underset{\sim}{2}}^{q}$ if $p=0$. Adopt the convention that $r S$ means the disjoint union of $r$ copies of $S$ for $r>0$ and for any poset $S$. Then

$$
H(F \mathscr{A}(s)) \cong \begin{cases}(2 p s) \mathbf{2}^{q s} & (p>0), \\ 2^{q s} & (p=0) .\end{cases}
$$

Remembering that the Priestley dual of $F \mathbf{D}(k)$ is ${\underset{2}{2}}^{k}$ for any $k$, we conclude that the D-reduct of $F \mathscr{A}(s)$ is $(F \mathbf{D}(q s))^{2 p s}$ if $p>0$, and $F \mathbf{D}(q s)$ if $p=0$ and $q=m=|N|$. Notice this result depends only on the parameters $p$ and $q$ and not on the semigroup structure of $N$. The latter gives the family of operations $\gamma_{\mu}$, and combines with the \pm assignment to determine the algebraic operations of $F \mathscr{A}(s)$.

4.15. Example. Involutive Stone algebras. The monoid $\mathbf{N}^{\mathrm{IS}}$ was given above, in Figure 7. Free algebras in IS and its subvarieties were first considered by Santos in [32]. We show how our general framework yields $F \mathbf{I S}(s)$. It is easily checked that the maps $\eta_{n}, \eta_{g}$ and $\eta_{g n}$ are order-preserving. Therefore Theorem 4.8 applies to IS $=\mathbb{U S P}(\underline{P})=\mathbb{H} \mathbb{S} \mathbb{P}(\underline{P})$, where $H(P)=\mathbf{N}^{\mathbf{I S}}$. We represent the elements of $P$ as 4-tuples $\left\langle a_{1}, a_{n}, a_{g}, a_{g m}\right\rangle$, with

$$
\begin{aligned}
& \gamma_{n}\left(\left\langle a_{1}, a_{n}, a_{g}, a_{g n}\right\rangle\right)=\left\langle a_{n}, a_{n}, a_{g n}, a_{g n}\right\rangle, \\
& \gamma_{g}\left(\left\langle a_{1}, a_{n}, a_{g}, a_{g n}\right\rangle\right)=\left\langle a_{g}, a_{n}, a_{1}, a_{g n}\right\rangle,
\end{aligned}
$$

and the \pm -order is given by

$$
\begin{aligned}
& \left\langle a_{1}, a_{n}, a_{g}, a_{g n}\right\rangle \preccurlyeq\left\langle b_{1}, b_{n}, b_{g}, b_{g n}\right\rangle \\
& \quad \text { if and only if } a_{1} \leqslant b_{1}, a_{n}=b_{n}, a_{g} \geqslant b_{g}, a_{g n}=b_{g n},
\end{aligned}
$$

as shown in Figure 11.

We see immediately from this that for $1 \leqslant s<\omega$,

$$
F \mathbf{I S}(s) \cong \prod_{k=0}^{s} F \mathbf{D}(2 k)^{\left(\begin{array}{c}
s \\
k
\end{array}\right)^{s-k}},
$$

where $F \mathbf{D}(2 k)$, qua involutive Stone algebra, is just the $k$-fold copower of the generating algebra $\underline{P}$.

4.16. Example. Kleene-Stone algebras. In this case the elements of $\underline{P}^{\mathbf{N}}$ are given by 4-tuples $\left\langle a_{g h}, a_{g}, a_{1}, a_{h}\right\rangle$, with $a_{g h} \leqslant a_{g} \leqslant a_{1} \leqslant a_{h}$. The operations $\gamma_{g}$ and $\gamma_{h}$ are given by

$$
\begin{aligned}
& \gamma_{g}\left(\left\langle a_{g h}, a_{g}, a_{1}, a_{h}\right\rangle\right)=\left\langle a_{g h}, a_{1}, a_{g}, a_{h}\right\rangle, \\
& \gamma_{h}\left(\left\langle a_{g h}, a_{g}, a_{1}, a_{h}\right\rangle\right)=\left\langle a_{g h}, a_{g h}, a_{h}, a_{h}\right\rangle .
\end{aligned}
$$




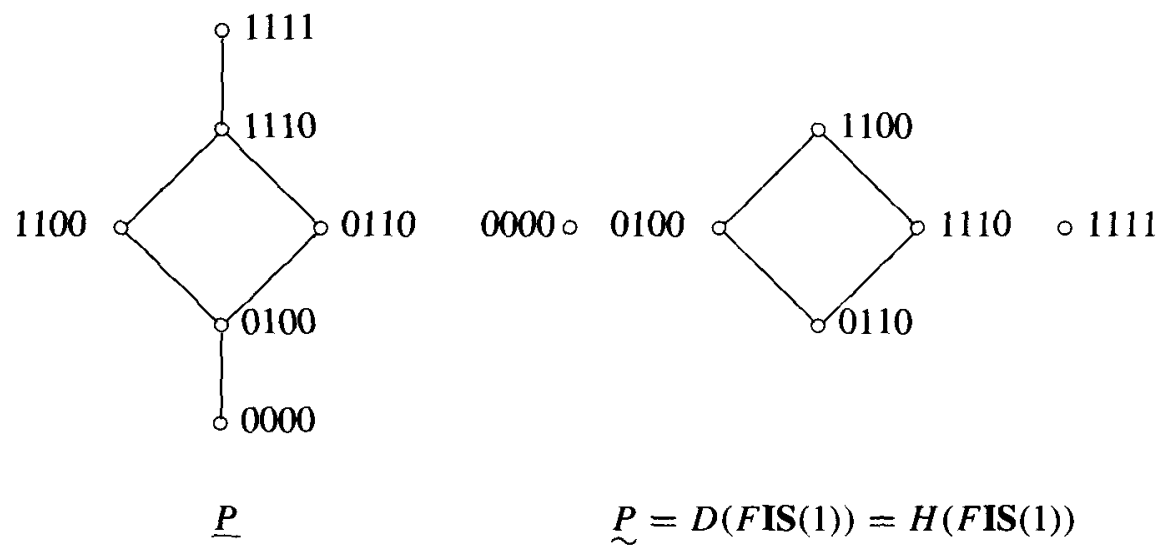

FIGURE 11

The \pm -order is given by

$$
\begin{aligned}
& \left\langle a_{g h}, a_{g}, a_{1}, a_{h}\right\rangle \preccurlyeq\left\langle b_{g h}, b_{g}, b_{1}, b_{h}\right\rangle \\
& \quad \text { if and only if } a_{g h}=b_{g h}, a_{g} \geqslant b_{g}, a_{1} \leqslant b_{1}, a_{h}=b_{h} .
\end{aligned}
$$

Because $\eta_{g}$ fails to be an endomorphism, we need a 2-sorted schizophrenic object, consisting of the sets of 4-element strings

$$
\begin{aligned}
& B=\{0000,0001,0101,0111,1111\}, \\
& g(B)=\{0000,0001,0011,0111,1111\} .
\end{aligned}
$$

With the two components drawn with their \pm -order, $B \cup \dot{\cup} g(B)$ is as shown in Figure 12.

An algebraic description of the free Kleene-Stone algebras was given in [23], and has also been obtained by Santos in [32]. We can see very easily from our monoid formalism how the free KS-algebras arise. The translation process applied to our 2sorted object simply glues the minimal points of the component $B$ to the corresponding maximal points of the component $g(B)$. This, made into a Kleene-Stone algebra dual space in the unique way possible, is $H(F \mathbf{K S}(1))$. If the isolated points 0000 and 1111 were not present, we should have just the structure giving the natural duality for Kleene algebras, so the $\mathbf{D}$-reducts related by

$$
F \mathbf{K S}(1)=F \mathbf{K}(1) \times 2^{2}
$$




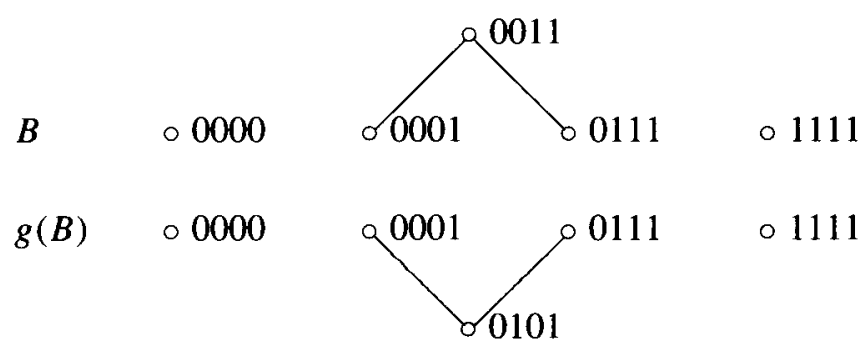

FIGURE 12. $D(F \mathbf{K S}(1))$

More generally we obtain $H(F \mathbf{K S}(s))$ by taking the $s$-fold products of the ordered sets $B$ and $g(B)$, glueing corresponding minimals and maximals. On each order component the map $g$ dual to the Kleene negation is the same as if we were dealing with the dual of a free Kleene algebras, while the map $h$ just takes each point to the unique maximal point above it. Since these maps are completely specified this way we consider just the underlying ordered sets. Let $P$ be the 2-element antichain, and $Q$ the 2-element antichain with a top element adjoined, so $B \cong P \cup Q$ and $g(B) \cong P \cup Q^{\delta}$. For a poset $S$ let $r S$ denote the disjoint union of $m$ copies of $S$, with the convention that $0 S$ means a 1-element chain. Then

$$
B^{s} \cong \bigcup_{0 \leqslant i \leqslant s}\left(\begin{array}{l}
s \\
i
\end{array}\right)\left(P^{s-i} \times Q^{i}\right)=\bigcup_{0 \leqslant i \leqslant s} 2^{s-i}\left(\begin{array}{l}
s \\
i
\end{array}\right) Q^{i}
$$

and dually, whence by the translation process,

$$
H(F \mathbf{K S}(s)) \cong \bigcup_{0 \leqslant i \leqslant s} 2^{s-i}\left(\begin{array}{l}
s \\
i
\end{array}\right) H(F \mathbf{K}(i))
$$

Dualising, we get

$$
F \mathbf{K S}(s) \cong \prod_{i=0}^{s}(F \mathbf{K}(i))^{2^{s-i}\left(\begin{array}{l}
s \\
i
\end{array}\right)},
$$

as Kleene algebras, with the Stone operation given by the pseudocomplement. The formula for the cardinality of $F \mathbf{K S}(s)$ derived from this is given in [23]; the algebra isomorphism is not given explicitly there. 


\section{References}

[1] M. E. Adams and H. A. Priestley, 'Equational bases for varieties of Ockham algebras', Algebra Universalis 32 (1994), 368-397.

[2] R. Balbes and Ph. Dwinger, Distributive lattices (University of Missouri Press, Columbia, 1974).

[3] T. S. Blyth, A. S. A. Noor and J. C. Varlet, 'Ockham algebras with de Morgan skeletons', J. Algebra $117(1988), 165-178$.

[4] _ , 'Equational bases for subvarieties of double MS-algebras', Glasgow Math. J. 31 (1989), 1-16.

[5] T. S. Blyth and J. C. Varlet, 'Subdirectly irreducible double MS-algebras', Proc. Roy. Soc. Edinburgh Sect. A 98 (1994), 241-247.

[6] _- Ockham algebras (Oxford University Press, Oxford, 1994).

[7] S. Burris and H. P. Sankappanavar, A course in universal algebra (Springer, New York, 1981).

[8] R. Cignoli and M. S. de Gallego, ' The lattice structure of some Łukasiewicz algebras', Algebra Universalis 13 (1981), 315-328.

[9] _ - 'Dualities for some De Morgan algebras with operators and Łukasiewicz algebras', $J$. Austral. Math. Soc. (Series A) 34 (1983), 377-393.

[10] D. M. Clark and B. A. Davey, Natural dualities for the working algebraist, in preparation (Cambridge University Press).

[11] W. H. Cornish, 'Monoids acting on distributive lattices', manuscript (invited talk at the annual meeting of the Austral. Math. Soc., May 1977).

[12] - Antimorphic action. Categories of algebraic structures with involutions or antiendomorphisms, R \& E Res. Exp. Math., 12 (Heldermann Verlag, Berlin, 1986).

[13] W. H. Cornish and P. R. Fowler, 'Coproducts of Kleene algebras', J. Austral. Math. Soc. (Series A) 27 (1979) , 209-220.

[14] B. A. Davey, 'On the lattice of subvarieties', Houston J. Math. 5 (1979), 183-192.

[15] — 'Duality theory on ten dollars a day', in: Algebras and orders (eds. I. G. Rosenberg and G. Sabidussi) NATO Advanced Study Institute Series, Series C, 389 (Kluwer, Dordrecht, 1993), $71-111$.

[16] B. A. Davey and H. A. Priestley, 'Lattices of homomorphisms', J. Austral. Math. Soc. (Series A) 40 (1986), 364-406.

[17] _ - 'Generalised piggyback dualities and applications to Ockham algebras', Houston J. Math. 13 (1987), 151-197.

[18] - Introduction to lattices and order (Cambridge University Press, Cambridge, 1990).

[19] B. A. Davey and H. Werner, 'Dualities and equivalences for varieties of algebras', in: Contributions to lattice theory (Szeged, 1980) (eds. A. P. Huhn and E. T. Schmidt) Colloq. Math. Soc. János Bolyai 33, (North-Holland, Amsterdam 1983),pp. 101-275.

[20] —_, 'Piggyback-dualitäten', Bull. Austral. Math. Soc. 32 (1985), 1-32.

[21] —_, 'Piggyback dualities', in: Colloq. Math. Soc. János Bolyai 43 (1986), 61-83.

[22] M. S. Goldberg, 'Distributive Ockham algebras: free algebras and injectivity', Bull. Austral. Math. Soc. 24 (1981) , 161-203.

[23] F. Guzman and C. C. Squier, 'Subdirectly irreducible and free Kleene-Stone algebras', Algebra Universalis 31 (1994), 266-273.

[24] H. A. Priestley, 'Ordered sets and duality for distributive lattices', in: Orders, description and roles (eds. M. Pouzet and D. Richard), Ann. Discrete Math. 23 (North-Holland, Amsterdam, 1984), pp. $39-60$.

[25] _ - 'The determination of the lattices of subvarieties of certain congruence-distributive varieties', Algebra Universalis 32 (1994) , 44-62.

[26] _ _ 'Natural dualities', in: Lattice theory and its applications - a volume in honor of Garrett 
Birkhoff's 80th birthday (eds. K. A. Baker and R. Wille) (Heldermann Verlag, Berlin, 1995), pp. 185-209.

[27] — 'A note on equational bases', Bull. Soc. Roy. Sci. Liège 64 (1995), 113-118.

[28] _ , 'Natural dualities for varieties of $n$-valued Łukasiewicz algebras', Studia Logica 54, 333370.

[29] - 'Dualities and identities I', preprint.

[30] H. A. Priestley and R. Santos, 'Dualities and identities II: DMS-algebras', preprint.

[31] —_, 'Varieties of distributive lattices with unary operations II', Portugal Math., to appear.

[32] R. Santos, 'Involutive Stone algebras and regular $\alpha$ De Morgan algebras', manuscript.

[33] _ - 'Natural dualities for some subvarieties of DMS', manuscript.

[34] H. P. Sankappanavar, 'Linked double weak Stone algebras', Z. Math. Logik Grundlag. Math. 35 (1989), 485-494.

[35] M. Sequeira, 'Double $M S_{n}$-algebras and double $K_{m . n}$-algebras', Glasgow Math. J. 35 (1993), 189-201.

[36] A. Urquhart, 'Lattices with a dual homomorphic operation', Studia Logica 38 (1979), 201-209.

[37] _ 'Lattices with a dual homomorphic operation II', Studia Logica 40 (1981) , 391-404.

[38] J. C. Varlet, 'MS-algebras: a survey', in: Lattices, semigroups and universal algebra (Lisbon, 1988) (eds. J. Almeida, G. Bordalo and Ph. Dwinger) (Plenum Press, New York, 1990),pp. 299313.

\section{Mathematical Institute}

24/29 St Giles

Oxford OXI 3LB

England

e-mail: hap@maths.ox.ac.uk 